\title{
Fundamental Sensitivity Limitations of Nanomechanical Resonant Sensors Due to Thermomechanical Noise
}

\author{
Alper Demir ${ }^{\circledR}$, Fellow, IEEE, and M. Selim Hanay
}

\begin{abstract}
Nanomechanical resonators are used as high performance sensors of physical stimuli such as force and mass changes. Any such physical stimulus produces a shift in the resonance frequency of the nanomechanical structure, which can be measured accurately by using a feedback system that locks the frequency of a signal generator to the resonance. Closed-loop frequency tracking is the most prevalent technique in the fields of nanomechanical sensors and non-contact atomic force microscopy. Ultimate performance of sensors is limited by various nonideal effects such as temperature variations, radiation, electromagnetic interference, and noise arising from inherent physical mechanisms. Here, we consider the noise performance of nanomechanical resonant sensors, which has so far eluded explanation with conflicting results reported in the literature. We present a precise theory for these ubiquitous sensors based on nanomechanical resonators under feedback in order to decipher the fundamental sensitivity limitations due to thermomechanical noise. The results we obtain, when the performance is limited by the thermomechanical noise of the resonator, are in complete agreement with the ones from stochastic simulations. Our findings shed light on recent results in the literature and resolve a critical problem regarding the frequency noise of nanomechanical sensors under feedback. Our results have applications in nanomechanics, atomic force microscopy, microwave and suspended microchannel resonators.
\end{abstract}

Index Terms - Nano-mechanical resonant sensor, phase-locked loop, frequency-locked loop, thermo-mechanical noise, Allan deviation.

\section{INTRODUCTION}

$\mathbf{S}$ TATE-OF-THE-ART nanomechanical resonators are used as high performance detectors, achieving yoctogram and single-protein resolutions in inertial mass sensing, owing to their ever diminishing size and superior frequency stability [1]-[5]. In the most prevalent resonant sensor configuration, a feedback system tracks the resonance frequency by driving the resonator with a signal generator that is locked to the resonance [2], [3], [6], [7]. Even though this feedback system is known as a Phase-Locked Loop (PLL) in the nanomechanical sensors community, it is, in fact, a Frequency-Locked Loop (FLL). Briefly, a PLL contains two oscillators, and locks a noisier oscillator to a high quality reference oscillator. On the

Manuscript received July 5, 2019; revised September 27, 2019; accepted October 17, 2019. Date of publication October 21, 2019; date of current version January 24,2020 . This work was supported by the Scientific and Technological Research Council of Turkey (TUBITAK) under Grant EEEAG-115E230. The associate editor coordinating the review of this article and approving it for publication was Prof. Sheng-Shian Li. (Corresponding author: Alper Demir.)

A. Demir is with the Department of Electrical Engineering, Koç University, 34450 Istanbul, Turkey (e-mail: aldemir@ku.edu.tr).

M. S. Hanay is with the Department of Mechanical Engineering, Bilkent University, 06800 Ankara, Turkey (e-mail: selimhanay@bilkent.edu.tr).

Digital Object Identifier 10.1109/JSEN.2019.2948681 other hand, an FLL contains only one oscillator, and locks the frequency of a signal generator (oscillator) to a reference resonator, precisely the case in nanomechanical sensors. FLLs have been in use for a long time in other disciplines, for instance, in passive atomic frequency standards since the 1960s, in order to lock the frequency of an oscillator to an atomic transition [8]. We use the proper term FLL instead of PLL in order to be consistent with the historical context and other disciplines. Most of the concepts and techniques we use in this paper are well known in the domains of precision oscillators, atomic frequency standards and frequency synthesis in electronics, but they have not been adequately applied to sensors. We build on the extensive body of work that was done in these other disciplines over the past sixty years.

Ultimate performance of sensors is limited by various nonideal effects including inherent fluctuations and noise [9]-[13]. Currently, there is great interest in understanding the fundamental sensitivity limitations of nanomechanical sensors due to noise [7], [10]-[17]. Recently, Roy et al. [7] proposed that one can improve sensor performance by increasing damping in the resonator, i.e., with resonators that have lower quality factors. This is diametrically opposed to the current understanding in the nanomechanical sensors field. It has been established a 


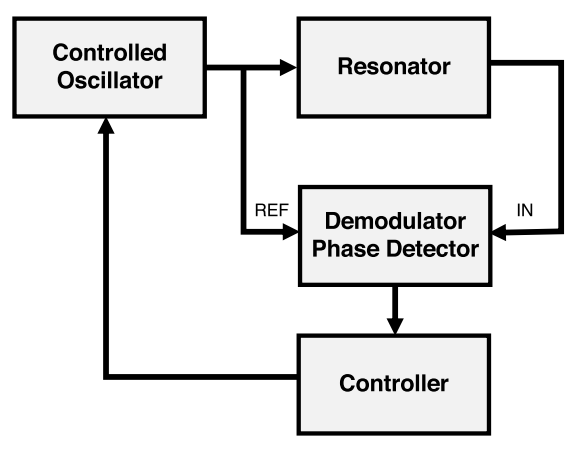

Fig. 1. Frequency-locked loop based resonant sensor.

long time ago in the frequency control field that phase noise is reduced with higher quality factors [18]-[20]. Roy et al. [7] offer a two-part argument as to how one may obtain better performance with a lower quality factor. First, they observe that nanomechanical resonators with lower quality factors can be driven harder before Duffing nonlinearity kicks in. The thermomechanical noise becomes dominant with higher drive strength and larger damping, enabling operation at a higher Signal-to-Noise-Ratio (SNR). Roy et al. operate their sensors at the onset of Duffing nonlinearity, which imposes the condition that SNR is inversely proportional to the quality factor $Q$. This first part of their proposal is in alignment with previous studies. Secondly, they claim that, with SNR $\propto$ $1 / Q$, one obtains much better performance than expected at a lower $Q$ with an FLL based architecture. Their explanation as to how this improvement arises at low $Q$ is based on a revelation that the phase noise spectrum flattens at low frequencies, in contrast with a common approximation used in high $Q$ cases. Although this claim involves a novel observation, it warrants further investigation. We investigate in detail whether one can obtain better performance with larger damping in an FLL based sensor. Our conclusion is in the negative. We arrive at this result by developing a general noise analysis framework for an FLL based sensor architecture as in Fig. 1, with details in Fig. 2. We use our analysis framework to precisely characterize the performance of an FLL based sensor, for different $Q$ values and feedback parameters, when the thermomechanical noise of the resonator is dominant. Our analysis framework can be used to assess the performance of FLL based sensors with other sources of noise, as well as for other FLL sensor configurations such as multi-mode [3] and nonlinear [21] sensing. The theory we develop is not specific to nanomechanics, it may be used for resonant sensors in other domains, for microwave resonators [22], [23] and atomic force microscopy [24]-[28].

The outline of the paper is as follows. We first present a theory overview in Section II providing a succinct account of the development, where we omit all derivations but point the reader to the more detailed treatment in Sections III and IV which can be skipped at first reading without loss of continuity. We present the models used for the FLL components in Section III. The detailed derivations and ancillary results are provided in Section IV. The results from our theory, comparisons with recent literature and stochastic simulations,

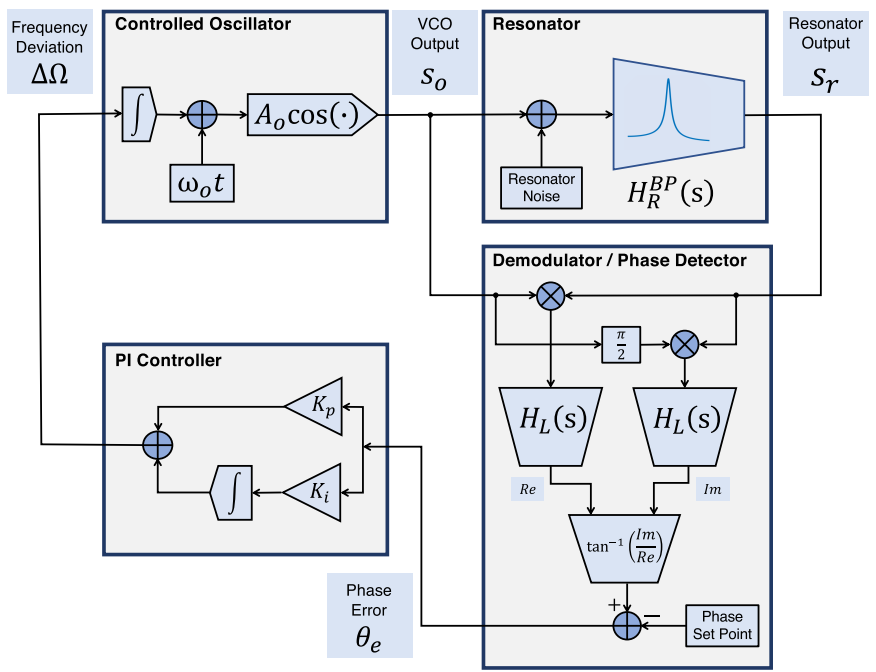

Fig. 2. Full model of FLL based resonator tracking. A voltage or numerically-controlled oscillator sets the frequency of the drive received by the resonator. In addition to drive, thermomechanical noise enters the bandpass transfer function $H_{R}^{B P}(\mathrm{~s})$ of the resonator. The demodulator/phase detector unit computes the phase difference between the response of the resonator and the drive signal. Resulting phase error $\theta_{e}$ is processed by the PI controller unit. Detailed explanations of all the units and various symbols are provided in Section III.

and a critical discussion are given in Section V. Conclusions are stated in Section VI. Two appendices provide derivations and details, regarding spectral characterization and filtering of cyclo-stationary random processes and stochastic simulations.

\section{THEORY OVERVIEW}

We consider a resonator that is modeled as a damped harmonic oscillator as follows [29]

$$
\frac{d^{2}}{d t^{2}} x+\Gamma \frac{d}{d t} x+\omega_{r}^{2} x=\frac{F(t)}{m}
$$

where $x$ is the displacement, $m$ is the mass, $F(t)$ represents a force excitation, $\omega_{r}$ is the resonance frequency, $\Gamma=\frac{\omega_{r}}{Q}$ is the damping rate with $Q$ as the quality factor. Based on the fluctuation-dissipation theorem, the thermomechanical noise of the resonator can be modeled as a white noise source (inputreferred, as in Fig. 2) with a (two-sided) spectral density

$$
\mathcal{S}_{t h m}(\omega)=2 m \Gamma k_{B} T
$$

where $k_{B}$ is Boltzmann's constant, and $T$ is temperature [11].

The FLL based tracking system shown in Fig. 2 contains signals with widely varying frequencies, as well as in different domains. The outputs of the signal generator, i.e., the controlled oscillator $(\mathrm{CO})$, and the resonator are at a high frequency (near $\omega_{r}$ ). On the other hand, the phase error signal (phase detector output) and the frequency deviation (controller output) are low-frequency signals, below the FLL bandwidth. The frequency separation between these signals is at least four orders of magnitude. The signal of interest is the frequency deviation, since it is used to track the resonance frequency. The challenge in analyzing the FLL system is to accurately characterize the slow dynamics of the frequency deviation signal while capturing the impact of 


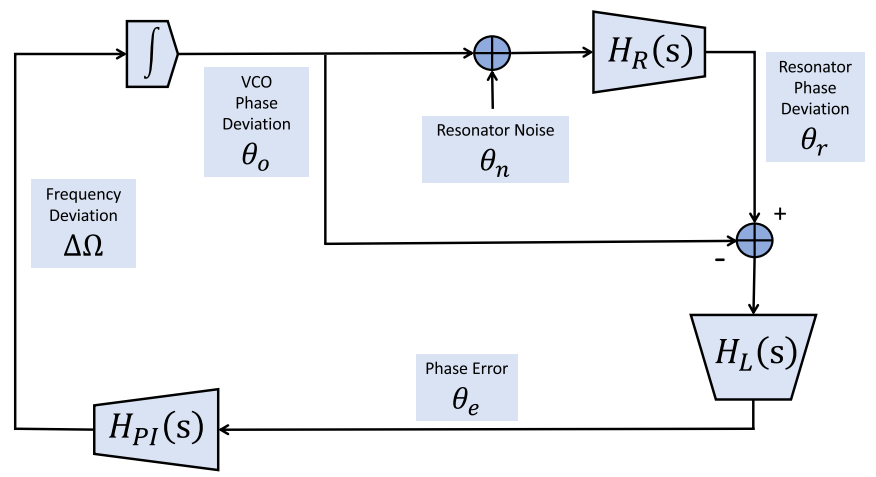

Fig. 3. Baseband equivalent model of FLL based resonant sensor.

the fast dynamics of the $\mathrm{CO}$ and the resonator in a correct manner. In order to accomplish this in a tractable manner, we have first developed a baseband (low-frequency) equivalent model of the CO-resonator-demodulator signal chain. The input and output of this chain of blocks are both low-frequency signals, whereas there is first low-to-high and then high-tolow frequency translation along the chain, and a nonlinear $\arctan (\cdot)$ operation at the end. This makes this composite system both nonlinear and time-varying. However, we were able to develop a Linear and Time-Invariant (LTI) model that is quite accurate, and verified it against numerical simulations of the full, nonlinear and time-varying system (Section IV-A). The baseband equivalent model for the (open-loop) signal chain from the frequency deviation $\Delta \Omega(t)$ to the phase error $\theta_{e}(t)$ can be expressed, in the Laplace domain, with $\Theta_{e}(\mathrm{~s})=$ $H_{L}$ (s) $H_{R}(\mathrm{~s}) \frac{1}{\mathrm{~s}} \Delta \Omega(\mathrm{s}) . H_{L}$ (s) is the transfer function of the low-pass filter in the demodulator, and $H_{R}(\mathrm{~s})=\frac{1}{1+\mathrm{s} \tau_{r}}$ with $\tau_{r}=\frac{2}{\Gamma}$ as the time-constant of the resonator, where $\mathbf{S}$ is the complex frequency variable. The open-loop model above yields to a baseband equivalent, phase domain model for the closed-loop FLL, as shown in Fig. 3, provided that $H_{L}(0)=1$ and the phase set point in the demodulator is $-\pi / 2$ to secure lock at resonance.

The model derived for the deterministic behavior needs to be augmented with a model for the noise dynamics. The nonlinear and time-varying nature of the system, and the nonstationary nature of the noise processes, makes this very challenging. We were able to model the entire noise dynamics by a simpler system, where an equivalent stationary noise process passes through baseband equivalent LTI filters (Section IV-B). This model, in the frequency domain, is summarized with

$$
\mathcal{S}_{\theta_{e}}(\omega)=\frac{4 m \omega_{r}^{2} k_{B} T}{A_{o}^{2} Q^{2} \Gamma}\left|H_{L}(j \omega)\right|^{2}\left|H_{R}(j \omega)\right|^{2}
$$

where $j^{2}=-1, A_{o}$ is the amplitude of the signal that drives the resonator, and $\mathcal{S}_{\theta_{e}}(\omega)$ is the power spectral density (PSD) of the phase error. Then,

$$
\mathcal{S}_{\theta_{n}}(\omega)=\frac{4 m \omega_{r}^{2} k_{B} T}{A_{o}^{2} Q^{2} \Gamma}=\frac{2 m \tau_{r} \omega_{r}^{2} k_{B} T}{A_{o}^{2} Q^{2}}
$$

is defined as the PSD of a white noise process $\theta_{n}(t)$, which represents the thermomechanical noise of the resonator in an input-referred manner. This noise goes through two filters,
$H_{R}$ (s) and $H_{L}$ (s), as in both equation (3) and Fig. 3, to produce the phase error noise.

With baseband equivalent models for both the deterministic and noise dynamics of the FLL components, we can proceed with the noise analysis of the closed-loop system based on Fig. 3 by writing the following equation

$\Theta_{o}(\mathrm{~s})=\frac{1}{\mathrm{~s}} H_{P I}(\mathrm{~s}) H_{L}(\mathrm{~s})\left[H_{R}(\mathrm{~s})\left[\Theta_{n}(\mathrm{~s})+\Theta_{o}(\mathrm{~s})\right]-\Theta_{o}(\mathrm{~s})\right]$.

Solving the above (Section IV-C) yields the transfer function from $\Theta_{n}(\mathrm{~s})$ to $\Delta \Omega(\mathrm{s})$

$$
H_{\theta_{n}}^{\Delta \Omega}(\mathrm{s})=\frac{1}{\tau_{r}}\left[\frac{\left(\mathrm{s} K_{p}+K_{i}\right) H_{L}(\mathrm{~s})}{\mathrm{s}^{2}+\frac{\mathrm{s}}{\tau_{r}}+\left(\mathrm{s} K_{p}+K_{i}\right) H_{L}(\mathrm{~s})}\right]
$$

and the PSD of $\Delta \Omega(t)$ is given by

$$
\mathcal{S}_{\Delta \Omega}(\omega)=\left|H_{\theta_{n}}^{\Delta \Omega}(j \omega)\right|^{2} \mathcal{S}_{\theta_{n}}(\omega)
$$

with $\mathcal{S}_{\theta_{n}}(\omega)$ as given in equation (4). The transfer function in equation (5) satisfies $H_{\theta_{n}}^{\Delta \Omega}(\mathrm{s} \rightarrow 0)=\frac{1}{\tau_{r}}$ and $H_{\theta_{n}}^{\Delta \Omega}(\mathrm{s} \rightarrow \pm \infty)=0 . H_{\theta_{n}}^{\Delta \Omega}(\mathrm{s})$ represents a low-pass filter, setting the loop bandwidth for the FLL. With $\mathcal{S}_{\theta_{n}}(\omega)$ representing a white spectrum, the frequency deviation $\Delta \Omega(t)$ has the characteristics of bandlimited (low-pass filtered) white noise. The phase deviation $\theta_{o}(t)$, the time-integral of $\Delta \Omega(t)$, has the characteristics of a random walk, albeit not in the form of a standard Brownian motion. We emphasize that this random walk aspect of phase deviation is not arising from the inherent phase noise of the $\mathrm{CO}$, which is assumed negligible due to the high precision of the signal generator. The random walk phase deviation here is due to thermomechanical noise circulating around the loop. The loop dynamics converts the additive noise of the resonator into phase noise in the $\mathrm{CO}$. Our analysis reveals precisely how this conversion occurs. The resultant phase noise in the $\mathrm{CO}$ ultimately limits the frequency tracking accuracy of the FLL and represents a fundamental limit on the sensitivity of the resonator based sensor system.

We characterize the frequency tracking accuracy of the FLL in terms of Allan Variance (AV) [30]-[34], which can be computed using

$$
\sigma_{y}^{2}(\tau)=\frac{4}{\pi \tau^{2}} \int_{-\infty}^{+\infty} \frac{\left[\sin \left(\frac{\omega \tau}{2}\right)\right]^{4}}{\omega^{2}} \frac{\mathcal{S}_{\Delta \Omega}(\omega)}{\omega_{r}^{2}} d \omega
$$

with $\mathcal{S}_{\Delta \Omega}(\omega)$ in equation (6) (Section IV-D). The integral above can not be evaluated analytically for all values of $\tau$. However, we can evaluate it numerically (results to be reported later in Fig. 5(a) and Fig. 6), and also obtain an analytical expression for an asymptote valid for large $\tau$

$$
\sigma_{y}^{2}(\tau)=\frac{m \omega_{r} k_{B} T}{A_{o}^{2} Q^{3}} \frac{1}{\tau}=\frac{1}{8(Q \mathrm{SNR})^{2} \mathrm{BW}} \frac{1}{\tau}
$$

where resonator Signal-to-Noise-Ratio (SNR) is defined as

$$
\mathrm{SNR}=\sqrt{\frac{\text { input signal power }}{\text { input noise power }}}=\sqrt{\frac{A_{o}^{2} Q}{8 m \omega_{r} k_{B} T \text { BW }}}
$$

with BW as the (one-sided) noise bandwidth, set to the bandwidth of the low-pass filters in the demodulator. 
The large- $\tau$ asymptote for AV with $1 / \tau$ dependence represents random walk phase noise [30]-[34]. For time scales larger than the loop time-constant, the resulting phase deviation, arising from the thermomechanical noise of the resonator and the loop dynamics, has a random walk nature. The result in equation (8) is independent of the loop and controller parameters (apart from an indirect dependence on them through BW) such as $K_{p}$ and $K_{i}$ and the particular choice for the filter transfer function $H_{L}(\mathrm{~s})$. On the other hand, these parameters do determine the loop bandwidth, and how AV changes with $\tau$ for short time scales within the loop time-constant.

\section{COMPONENT MODELS}

\section{A. Resonator}

The resonator, modeled as a damped harmonic oscillator, is described by equation (1). The damping rate

$$
\Gamma=\frac{\omega_{r}}{Q}
$$

determines the line-width of the resonator's frequency response (from input $F(t)$ to output $x(t)$ ), which is given by

$$
H_{R}^{B P}(\mathrm{~s})=\frac{X(\mathrm{~s})}{F(\mathrm{~s})}=\frac{1}{m} \frac{1}{\mathrm{~s}^{2}+\Gamma \mathrm{s}+\omega_{r}^{2}} .
$$

If the thermomechanical noise, modeled by the PSD in (2), is the only input to the resonator, the mean kinetic energy of the resonator can be computed as follows

$$
E_{K}=\mathbf{E}\left[\frac{1}{2} m\left(\frac{d}{d t} x\right)^{2}\right]
$$

where $\mathbf{E}[\cdot]$ denotes expectation, which can be computed using the Wiener-Khinchin theorem as below

$$
E_{K}=\frac{1}{2} m \frac{1}{2 \pi} \int_{-\infty}^{\infty} \omega^{2} \mathcal{S}_{x}(\omega) d \omega
$$

where $\mathcal{S}_{x}(\omega)$ is the PSD of the resonator displacement $x(t)$ :

$$
\mathcal{S}_{x}(\omega)=\left|H_{R}^{B P}(j \omega)\right|^{2} \mathcal{S}_{t h m}(\omega) .
$$

The integral in (13) yields $E_{K}=\frac{k_{B} T}{2}$ [35], consistent with the equipartition theorem of statistical mechanics [36].

\section{B. Demodulator}

The demodulator shown in Fig. 2 performs as a phase (difference) detector. The $\mathrm{CO}$ signal and the resonator output can be expressed as

$$
\begin{aligned}
& s_{o}(t)=A_{o} \cos \left(\omega_{o} t+\theta_{o}(t)\right), \\
& s_{r}(t)=A_{r} \cos \left(\omega_{r} t+\theta_{r}(t)\right) .
\end{aligned}
$$

We can express the operations in the in-phase and quadrature arms of the demodulator in a compact manner using complex arithmetic. We express the resonator output as

$$
s_{r}(t)=\frac{A_{r}}{2}\left[e^{j\left(\omega_{r} t+\theta_{r}(t)\right)}+e^{-j\left(\omega_{r} t+\theta_{r}(t)\right)}\right] .
$$

Then, the signals at the output(s) of the multipliers in the demodulator are the real and imaginary parts of

$$
\begin{gathered}
\frac{A_{r} A_{o}}{2}\left[e^{j\left(\omega_{r} t+\theta_{r}(t)\right)}+e^{-j\left(\omega_{r} t+\theta_{r}(t)\right)}\right] e^{-j\left(\omega_{o} t+\theta_{o}(t)\right)} \\
=\frac{A_{r} A_{o}}{2}\left[e^{j\left(\left(\omega_{r}-\omega_{o}\right) t+\theta_{r}(t)-\theta_{o}(t)\right)}\right. \\
\left.+e^{-j\left(\left(\omega_{r}+\omega_{o}\right) t+\theta_{r}(t)+\theta_{o}(t)\right)}\right] .
\end{gathered}
$$

We assume that the low-pass filters in the demodulator (shown as $H_{L}(\mathrm{~s})$ ) block the high-frequency, second term and pass the low-frequency, first term above. Thus, the outputs of the filters are given by (the real/imaginary parts of)

$$
\frac{A_{r} A_{o}}{2} e^{j\left(\left(\omega_{r}-\omega_{o}\right) t+\theta_{r}(t)-\theta_{o}(t)\right)} .
$$

Finally, the output of the $\arctan (\cdot)$ block is the phase difference between the resonator output and the $\mathrm{CO}$ signal

$$
\theta_{e}(t)=\left(\omega_{r}-\omega_{o}\right) t+\theta_{r}(t)-\theta_{o}(t)
$$

where we assume that $\omega_{r} \approx \omega_{o}$ and $\theta_{r}(t)-\theta_{o}(t)$ is a lowfrequency signal. In the actual model of the system, we do take into account the non-ideal nature of the low-pass filter $H_{L}(\mathrm{~s})$. The attenuation of the high-frequency component in (17) by a practical $H_{L}$ (s) is quite good due to the large frequency separation, whereas the low-frequency component does get modified by the filter, which we take into account in the analytical theory we develop further below. The phase set point in the demodulator is needed in order to keep the resonator at its resonance, as we show later.

\section{Controller}

We use a proportional-integral PI controller, as shown in Fig. 2, with a transfer function

$$
H_{P I}(\mathrm{~s})=K_{p}+\frac{K_{i}}{\mathrm{~s}} \text {. }
$$

We discuss later how to choose the controller parameters $K_{p}$ and $K_{i}$. The input to the controller is the phase error from the demodulator and its output is fed to the $\mathrm{CO}$, determining its frequency deviation from the nominal value $\omega_{o}$.

\section{Controlled Oscillator}

The controlled oscillator, i.e., the signal generator, is an essential component of the FLL. In an all-analog FLL system, it would be instantiated as a high precision analog voltagecontrolled oscillator (VCO), typically including a crystal as a time reference. In FLL tracking systems for NEMS applications, lock-in amplifiers (LIA) are routinely employed. In recent LIA based systems, the $\mathrm{CO}$ is digitally implemented on a DSP processor, as what is called a numerically controlled oscillator (NCO). The frequency precision of the NCO is then determined by the time base of the DSP, which may be locked to an atomic reference. We model the $\mathrm{VCO} / \mathrm{NCO}$ output with

$$
s_{o}(t)=A_{o} \cos \left(\omega_{o} t+\int^{t} \Delta \Omega\left(t^{\prime}\right) d t^{\prime}\right)
$$

where the frequency deviation $\Delta \Omega(t)$ is the control signal produced by the PI controller. Due to the high precision of the 


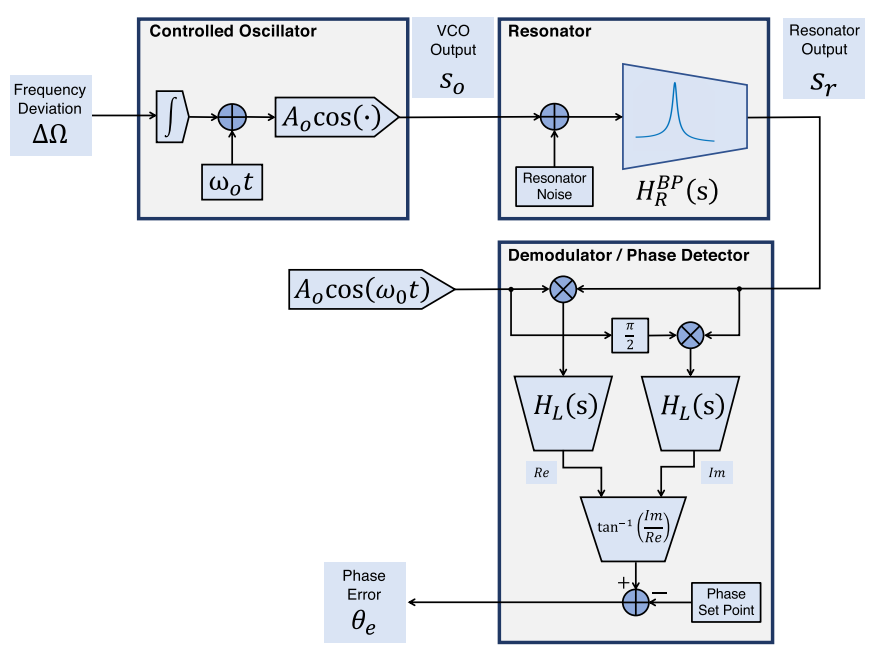

Fig. 4. Open-loop controlled oscillator-resonator-demodulator chain.

VCO or the time base of the NCO, we assume that the phase noise contribution of the $\mathrm{CO}$ is negligible. However, both the theory and the stochastic simulator that we have developed can be modified to include the phase noise contribution from the $\mathrm{CO}$, as well as noise from other components. Our main goal in this paper is to decipher the fundamental sensitivity limitation due to thermomechanical noise of the resonator.

\section{TheORY: DERIVATIONS AND DETAILS}

\section{A. Baseband Equivalent Phase Domain Model for the Resonator}

We consider the open-loop CO-resonator-demodulator chain shown in Fig. 4. In constructing this open-loop model, not only we have disconnected the main FLL loop but also we no longer feed the second demodulator input with the signal from the CO. Instead, this input is set to a sinusoidal signal at a constant frequency. In the final model we construct, we do take into account the fact that the second demodulator input is in fact set to the CO output. In the development below, we first set the resonator noise to zero and derive a model for the deterministic dynamics. Then, we also consider the resonator noise and include it in the final model.

We now walk through the signal chain, from the input (frequency deviation) $\Delta \Omega(t)$ to the output (phase error) $\theta_{e}(t)$. The phase deviation of the $\mathrm{CO}, \theta_{o}(t)$ is related to the frequency deviation with an integral

$$
\theta_{o}(t)=\int^{t} \Delta \Omega\left(t^{\prime}\right) d t^{\prime} .
$$

The output of the $\mathrm{CO}$ is given by

$$
\begin{aligned}
s_{o}(t) & =A_{o} \cos \left(\omega_{o} t+\theta_{o}(t)\right) \\
& =\frac{A_{o}}{2}\left[e^{j\left(\omega_{o} t+\theta_{o}(t)\right)}+e^{-j\left(\omega_{o} t+\theta_{o}(t)\right)}\right] .
\end{aligned}
$$

The operation above constitutes a low-to-high frequency translation, from $\theta_{o}(t)$ to $s_{o}(t)$. We consider only the first term on the second line of (23). As discussed in Section III-B, the signal that arises from the second term down the resonatordemodulator signal chain will be eventually blocked by the low-pass filter $H_{L}$ (s). The input to the resonator is given by

$$
s_{i n r}(t)=\frac{A_{o}}{2} e^{j\left(\omega_{o} t+\theta_{o}(t)\right)}=\frac{A_{o}}{2} e^{j \omega_{o} t} e^{j \theta_{o}(t)} .
$$

In order to compute the effect of the resonator on the signal, we move to frequency domain, by computing the Laplace transform (bilateral) of $s_{i n r}(t)$ above

$$
S_{i n r}(\mathrm{~s})=\mathcal{L}\left\{\frac{A_{o}}{2} e^{j \omega_{o} t} e^{j \theta_{o}(t)}\right\}=S_{i n r}^{B B}\left(\mathrm{~s}-j \omega_{o}\right)
$$

where

$$
S_{\text {inr }}^{B B}(\mathrm{~s})=\mathcal{L}\left\{\frac{A_{o}}{2} e^{j \theta_{o}(t)}\right\}=\mathcal{L}\left\{s_{\text {inr }}^{B B}(t)\right\} .
$$

While $s_{\text {inr }}(t)$ is a high-frequency, passband signal with its power concentrated around $\omega_{o}$ in the frequency domain, $s_{i n r}^{B B}(t)$ is a baseband, low-frequency signal with its power concentrated around zero frequency. When the FLL is tracking the resonance, the $\mathrm{CO}$ center frequency $\omega_{o}$ would be nominally equal to the resonance frequency $\omega_{r}$. If there is any resonance frequency shift, FLL would compensate for this by adjusting the frequency deviation $\Delta \Omega(t)$, and accordingly the phase deviation $\theta_{o}(t)$, of the CO. Thus, without loss of generality, we assume $\omega_{o}=\omega_{r}$ as we proceed. We compute the output of the resonator with its input as in (25)

$$
S_{\text {outr }}(\mathrm{s})=H_{R}^{B P}(\mathrm{~s}) S_{\text {inr }}(\mathrm{s})
$$

where $H_{R}^{B P}$ (s) is the resonator transfer function in (11). $H_{R}^{B P}(\mathrm{~s})$ has a passband characteristics centered around $\omega_{o}$. Thus, as the input $s_{i n r}(t)$ is a passband signal centered around the same frequency, so is the output $s_{\text {outr }}(t)$. Hence, we have

$$
\begin{aligned}
& s_{\text {outr }}(t)=e^{j \omega_{o} t} s_{\text {outr }}^{B B}(t) \\
& S_{\text {outr }}(\mathrm{s})=S_{\text {outr }}^{B B}\left(\mathrm{~s}-j \omega_{o}\right)
\end{aligned}
$$

where $s_{\text {outr }}^{B B}(t)$ is a baseband, low-frequency signal with its power concentrated around zero frequency. Combining (25), (27) and (29), we obtain

$$
\begin{aligned}
S_{\text {outr }}^{B B}\left(\mathrm{~s}-j \omega_{o}\right) & =H_{R}^{B P}(\mathrm{~s}) S_{\text {inr }}^{B B}\left(\mathrm{~s}-j \omega_{o}\right) \\
S_{\text {outr }}^{B B}(\mathrm{~s}) & =H_{R}^{B P}\left(\mathrm{~s}+j \omega_{o}\right) S_{\text {inr }}^{B B}(\mathrm{~s})
\end{aligned}
$$

We define

$$
H_{R}^{B B}(\mathrm{~s})=H_{R}^{B P}\left(\mathrm{~s}+j \omega_{o}\right)
$$

as the baseband equivalent transfer function of the resonator

$$
H_{R}^{B B}(j \omega)=\frac{1}{m} \frac{1}{-\left(\omega+\omega_{o}\right)^{2}+\frac{\omega_{o}}{Q} j\left(\omega+\omega_{o}\right)+\omega_{o}^{2}}
$$

where we used (11) and (31), and substituted $s=j \omega$ and $\Gamma=\omega_{o} / Q \cdot H_{R}^{B B}(j \omega)$ can be used as a baseband equivalent model for the resonator. An approximate (first-order) form for $H_{R}^{B B}(j \omega)$ (second-order) that we derive below simplifies the rest of our derivations considerably. We manipulate (32) (by combining the first and third terms in the denominator of the $\omega$ dependent part) to obtain

$$
H_{R}^{B B}(j \omega)=\frac{1}{m} \frac{1}{-\omega\left(\omega+2 \omega_{o}\right)+j \frac{\omega_{o}}{Q}\left(\omega+\omega_{o}\right)} .
$$


We note that $\omega$ in (33) above is small when compared with $\omega_{o}$. This is due to the frequency shift operation represented by (31). In the passband model of the resonator represented by $H_{R}^{B P}(j \omega)$, we have $\omega \approx \omega_{o}$, whereas in the baseband equivalent model represented by $H_{R}^{B B}(j \omega)=H_{R}^{B P}\left(j\left(\omega+\omega_{o}\right)\right)$, we have $\omega \approx 0$. We assume that $\omega \ll \omega_{o}$ in (33) and use the following approximations due to baseband nature of $H_{R}^{B B}(j \omega)$

$$
\omega+2 \omega_{o} \approx 2 \omega_{o}, \omega+\omega_{o} \approx \omega_{o} .
$$

The above can be interpreted as a sort of high- $Q$ approximation, but our goal is to develop a theory that is valid even for low- $Q$ resonators. We verified against simulations (which do not incorporate any approximations) that the baseband resonator model based on the above approximations remains accurate for a $Q$ that is as low as 10. With the above approximations, $H_{R}^{B B}(j \omega)$ can be simplified as follows

$$
\begin{aligned}
H_{R}^{B B}(j \omega) & \approx \frac{1}{m} \frac{1}{-2 \omega \omega_{o}+j \frac{\omega_{o}^{2}}{Q}} \\
& =\frac{Q}{m \omega_{o}^{2}} e^{-j \frac{\pi}{2}} \frac{1}{1+j \omega \frac{2 Q}{\omega_{o}}} .
\end{aligned}
$$

Finally, $H_{R}^{B B}(j \omega)$ can be represented as a Laplace transform:

$$
H_{R}^{B B}(\mathrm{~s})=\frac{Q}{m \omega_{o}^{2}} e^{-j \frac{\pi}{2}} \frac{1}{1+\mathrm{s} \frac{2 Q}{\omega_{o}}} .
$$

We define the resonator time constant with

$$
\tau_{r}=\frac{2 Q}{\omega_{o}}=\frac{2}{\Gamma}
$$

and obtain

$$
H_{R}^{B B}(\mathrm{~s})=\frac{Q}{m \omega_{o}^{2}} e^{-j \frac{\pi}{2}} \frac{1}{1+\mathrm{s} \tau_{r}} .
$$

The above is essentially a first-order, one-pole, low-pass transfer function, with a DC gain and an extra phase shift. If the input to the resonator is a pure tone at the resonance frequency $\omega_{o}$ (corresponding to $s=0$ in (38)), then the steadystate output (also a pure tone at the same frequency) will have a $-\pi / 2$ phase shift with respect to the input.

We note that a resonator model as in (38) was derived in [6] using a time-domain response approach. Our treatment above based on the use of the baseband equivalent transfer function streamlines the model development process and reveals the exact nature of the approximations involved. The baseband equivalent representation for bandpass signals is commonly used in the analysis of communication systems [37]. This technique is similar to the ones used in other disciplines, known as complex amplitude representation for slow dynamics [14], and slowly varying envelope approximation [38].

Next, we move along the signal chain and characterize the impact of the demodulator. The demodulator features high-tolow frequency translation, undoing the low-to-high frequency translation by the $\mathrm{CO}$. The signals at the output(s) of the multipliers in the demodulator are the real/imaginary parts of

$$
s_{m}(t)=A_{o} e^{-j \omega_{o} t} s_{\text {outr }}(t)
$$

We substitute (28) into the above equation to obtain

$$
s_{m}(t)=A_{o} e^{-j \omega_{o} t} e^{j \omega_{o} t} s_{\text {outr }}^{B B}(t)=A_{o} s_{\text {outr }}^{B B}(t) .
$$

The demodulator extracts the baseband equivalent, complexvalued $s_{\text {outr }}^{B B}(t)$. It is further processed through the low-pass filter $H_{L}(\mathrm{~s})$, which nominally does not modify it. However, the filter is required in order to remove the high-frequency signals (at the outputs of the multipliers) that arise from the second term in (23), which we have ignored upfront.

The phase angle of the complex-valued output of the lowpass filters is produced with the $\arctan (\cdot)$ block, with inputs set to be the (real and imaginary parts of)

$$
s_{d}(t)=A_{d}(t) e^{j \theta_{d}(t)}
$$

where $A_{d}(t)$ is the amplitude and $\theta_{d}(t)$ is the phase. The output $\theta_{e}(t)$ of the $\arctan (\cdot)$ block is simply the phase $\theta_{d}(t)$. Then, we have

$$
\begin{aligned}
S_{d}(\mathrm{~s}) & =\mathcal{L}\left\{A_{d}(t) e^{j \theta_{d}(t)}\right\} \\
& =A_{o} H_{L}(\mathrm{~s}) H_{R}^{B B}(\mathrm{~s}) S_{i n r}^{B B}(\mathrm{~s}) \\
& =\frac{A_{o}^{2}}{2} H_{L}(\mathrm{~s}) H_{R}^{B B}(\mathrm{~s}) \mathcal{L}\left\{e^{j \theta_{o}(t)}\right\} .
\end{aligned}
$$

Thus, we have obtained a compact and simple model for the entire signal chain from the phase deviation $\theta_{o}(t)$ of the $\mathrm{CO}$ to the phase error $\theta_{e}(t)$, output of the phase detector. In doing so, we were able to capture everything with low-frequency signals, in a baseband equivalent manner. The model in (42) does not have any explicit frequency translation operations, making it time-invariant. However, this model is still nonlinear due to the phase-to-complex conversion, i.e., $e^{j}$, in the $\mathrm{CO}$, and the complex-to-phase conversion, i.e., $\arctan (\cdot)$, in the demodulator. Next, we introduce a further approximation and linearize this model.

We observe that the $\arctan (\cdot)$ block makes any scaling or DC gain factor up to that point along the signal chain irrelevant, i.e., the factor $A_{o} / 2$ in (26), the DC gain $Q /\left(m \omega_{o}^{2}\right)$ in (38), any DC gain in $H_{L}$ (s), and the factor $A_{o}$ in (40) are all immaterial for the final output of the demodulator. The final operation in the demodulator subtracts the phase set point from the computed phase, and is set to $-\pi / 2$ due to the phase shift in (38) due to the resonator. (The $-\pi / 2$ phase set point is, not related to, and distinct from the $\pi / 2$ phase shift applied to the $\mathrm{CO}$ signal for the quadrature arm of the demodulator.) We assume that $H_{L}(\mathrm{~s})$ does not introduce any extra phase shift for (complex-valued) DC signals. Then, if the phase deviation $\theta_{o}(t)$ of the $\mathrm{CO}$ is time-invariant, set to a constant as $\theta_{o}(t)=\theta_{c}$, then the final output of the demodulator, i.e., the phase error $\theta_{e}(t)$, is equal to this constant phase $\theta_{c}$. Thus, we remove all scaling factors, DC gains, as well as the $-\pi / 2$ phase shift in resonator, from the signal path, without changing the final phase error output of the demodulator. We define

$$
H_{R}(\mathrm{~s})=\frac{1}{1+\mathrm{s} \tau_{r}}
$$

which was obtained from (38) by removing the DC gain and the $-\pi / 2$ phase shift. We assume that the low-pass filter 
$H_{L}$ (s) has a DC gain of 1 and introduces no phase shift for DC signals, i.e., $H_{L}(0)=1$. We then modify (42) to obtain

$$
\mathcal{L}\left\{A_{d}(t) e^{j \theta_{d}(t)}\right\}=H_{L}(\mathrm{~s}) H_{R}(\mathrm{~s}) \mathcal{L}\left\{e^{j \theta_{o}(t)}\right\} .
$$

We next assume that the phase deviation $\theta_{o}(t)$ is small enough so that we can use the following approximation

$$
e^{j \theta_{o}(t)} \approx 1+j \theta_{o}(t) .
$$

The constant DC factor 1 above (real part) will go through $H_{R}(\mathrm{~s})$ and $H_{L}(\mathrm{~s})$ unmodified since $H_{L}(0)=H_{R}(0)=1$, whereas $j \theta_{o}(t)$ will be modified, producing

$$
1+j \theta_{d}(t) \approx e^{j \theta_{d}(t)}
$$

where

$$
1+j \mathcal{L}\left\{\theta_{d}(t)\right\}=1+j H_{L}(\mathrm{~s}) H_{R}(\mathrm{~s}) \mathcal{L}\left\{\theta_{o}(t)\right\} .
$$

Thus, the phase error $\theta_{e}(t)=\theta_{d}(t)$ (ignoring $-\pi / 2$ phase set point) can be computed with

$$
\begin{aligned}
\Theta_{e}(\mathrm{~s})=\mathcal{L}\left\{\theta_{e}(t)\right\} & =H_{L}(\mathrm{~s}) H_{R}(\mathrm{~s}) \mathcal{L}\left\{\theta_{o}(t)\right\} \\
& =H_{L}(\mathrm{~s}) H_{R}(\mathrm{~s}) \Theta_{o}(\mathrm{~s}) .
\end{aligned}
$$

Since $\Theta_{o}(\mathrm{~s})=\frac{1}{\mathrm{~s}} \Delta \Omega(\mathrm{s})$, we have

$$
\Theta_{e}(\mathrm{~s})=H_{L}(\mathrm{~s}) H_{R}(\mathrm{~s}) \frac{1}{\mathrm{~s}} \Delta \Omega(\mathrm{s}) \text {. }
$$

Thus, we have obtained a linear and time-invariant model for the entire signal chain, from the frequency deviation $\Delta \Omega(t)$ to the phase error $\theta_{e}(t)$.

In deriving the model above, we assumed that the second demodulator input is simply set to $A_{o} \cos \left(\omega_{0} t\right)$, as in the openloop model in Fig. 4. In the closed-loop FLL, this input is in fact set to the output of the $\mathrm{CO}$, making the demodulator effectively a phase difference detector between the resonator and the $\mathrm{CO}$ outputs. We now construct a baseband equivalent, phase domain model for the closed-loop FLL, as shown in Fig. 3, by taking this into account. In this model, the PI controller is represented as a transfer function $H_{P I}(\mathrm{~s})$ given in (20). We have verified all of the approximations we have performed in deriving the phase domain model against simulations of the full system model. Finally, we emphasize that, for the model in Fig. 3 to be valid, the baseband equivalent resonator transfer function $H_{R}$ (s) and the demodulator low-pass filter transfer function $H_{L}$ (s) need to satisfy $H_{L}(0)=H_{R}(0)=1$.

\section{B. Baseband Equivalent Noise Model for the Resonator}

Having derived a simple model for the deterministic dynamics of the system, we now turn to doing the same for the noise dynamics. We consider the open-loop system in Fig. 4 and initially set the $\mathrm{CO}$ output to zero. Thus, the resonatordemodulator chain is driven by only the resonator noise source, modeled as a stationary random process with a PSD as given in (2). This white noise source is shaped by the resonator, turning into a colored noise process with a passband PSD, but still stationary. In the demodulator, it goes through the two mixers (multipliers), turning into cyclo-stationary noise processes [39]-[41]. As explained later, the low-pass filters in the demodulator not only block the highfrequency parts but also stationarize these cyclo-stationary noise processes by removing the high-order cyclo-stationary components [39]-[41]. Finally, the noise processes in the in-phase and quadrature arms of the demodulator converge at the $\arctan (\cdot)$ nonlinearity, which performs a real/imaginary-tophase conversion, yielding a phase error noise process at the very end. The phase set point subtraction is a DC operation, and was taken into account as part of the deterministic dynamics of the system considered before. As summarized here, the thermomechanical noise of the resonator goes through a nonlinear and time-varying system with inherent frequency translation operations. However, as we did for the deterministic dynamics, we will be able to model the entire noise dynamics as captured by a much simpler system, where an equivalent stationary noise process passes through baseband equivalent linear and time-invariant filters.

We follow the noise path in Fig. 4 starting from the input of the resonator. With the PSD of the noise source at the resonator input as in (2), the noise PSD at the resonator output can be computed as follows

$$
\begin{aligned}
\mathcal{S}_{r}(\omega) & =\left|H_{R}^{B P}(j \omega)\right|^{2} \mathcal{S}_{t h m}(\omega) \\
& =\frac{2 \Gamma k_{B} T}{m} \frac{1}{\left(\omega^{2}-\omega_{o}^{2}\right)^{2}+\Gamma^{2} \omega^{2}} .
\end{aligned}
$$

Next, this passband stationary noise process is fed into the two multipliers that generate cyclo-stationary noise, which can not be characterized with a simple PSD. For cyclostationary processes, the PSD is a function of two variables, the frequency $\omega$ and time $t$, i.e., $\mathcal{S}_{c y c}(t, \omega)$, where the $t$ dependence is periodic and can be represented with a Fourier series as discussed in Appendix A, and [39]-[41]. The noise signal at the output of the in-phase multiplier is given by $s_{m R e}(t)=A_{o} \cos \left(\omega_{o} t\right) s_{r}(t)$ where $s_{r}(t)$ is the stationary noise signal at the output of the resonator with the PSD in (50). Then, the cyclic spectra of $s_{m R e}(t)$, as shown in Appendix $\mathrm{A}$, is given by

$$
\begin{aligned}
& \mathcal{S}_{m R e}^{(0)}(\omega)=\frac{A_{o}^{2}}{4}\left[\mathcal{S}_{r}\left(\omega-\omega_{o}\right)+\mathcal{S}_{r}\left(\omega+\omega_{o}\right)\right] \\
& \mathcal{S}_{m R e}^{(2)}(\omega)=\mathcal{S}_{m R e}^{(-2)}(\omega)=\frac{A_{o}^{2}}{4} \mathcal{S}_{r}(\omega) \\
& \mathcal{S}_{m R e}^{(k)}(\omega)=0 \text { for all other } k .
\end{aligned}
$$

The noise signal at the output of the quadrature multiplier is given by $s_{m I m}(t)=-A_{o} \sin \left(\omega_{o} t\right) s_{r}(t)$. The cyclic spectra of $s_{m I m}(t)$ is

$$
\begin{aligned}
& \mathcal{S}_{m I m}^{(0)}(\omega)=\frac{A_{o}^{2}}{4}\left[\mathcal{S}_{r}\left(\omega-\omega_{o}\right)+\mathcal{S}_{r}\left(\omega+\omega_{o}\right)\right] \\
& \mathcal{S}_{m I m}^{(2)}(\omega)=\mathcal{S}_{m I m}^{(-2)}(\omega)=-\frac{A_{o}^{2}}{4} \mathcal{S}_{r}(\omega) \\
& \mathcal{S}_{m I m}^{(k)}(\omega)=0 \text { for all other } k .
\end{aligned}
$$

The cyclo-stationary noise signals $s_{m R e}(t)$ and $s_{m I m}(t)$ are filtered with the low-pass filter $H_{L}(\mathrm{~s})$. As shown in Appendix A, this filter stationarizes these cyclo-stationary noise 
processes, and at the same time removes high-frequency components [40]. The stationary noise signals, $s_{d R e}(t)$ and $s_{d I m}(t)$, at the output of these filters have the following PSD

$$
\begin{aligned}
\mathcal{S}_{d R e}(\omega) & =\mathcal{S}_{d I m}(\omega) \\
& =\frac{A_{o}^{2}}{4}\left|H_{L}(j \omega)\right|^{2}\left[\mathcal{S}_{r}\left(\omega-\omega_{o}\right)+\mathcal{S}_{r}\left(\omega+\omega_{o}\right)\right]
\end{aligned}
$$

where $\mathcal{S}_{r}(\omega)$ is the PSD in (50). We next analyze the noise folding (Appendix A) and filtering represented by (53). $\mathcal{S}_{r}(\omega)$ is a passband, two-sided PSD with power concentrated around $\pm \omega_{o}$. Thus, $\mathcal{S}_{r}\left(\omega-\omega_{o}\right)$ has power concentrated around 0 and $2 \omega_{o}$, whereas for $\mathcal{S}_{r}\left(\omega+\omega_{o}\right)$ it is around 0 and $-2 \omega_{o}$. Assuming that $H_{L}(j \omega)$ is a low-pass filter with an effective bandwidth that is much less than $\omega_{o}$, satisfying $H_{L}\left( \pm j 2 \omega_{o}\right) \approx 0$, it will remove the noise component at $2 \omega_{o}$ in $\mathcal{S}_{r}\left(\omega-\omega_{o}\right)$ and the one at $-2 \omega_{o}$ in $\mathcal{S}_{r}\left(\omega+\omega_{o}\right)$. The noise components of interest are the ones around zero frequency. We evaluate these components as follows, based on (50):

$$
\begin{aligned}
\mathcal{S}_{r}(\omega)=\frac{2 \Gamma k_{B} T}{m} \frac{1}{\left[\left(\omega+\omega_{o}\right)\left(\omega-\omega_{o}\right)\right]^{2}+\Gamma^{2} \omega^{2}} \\
\mathcal{S}_{r}\left(\omega-\omega_{o}\right)+\mathcal{S}_{r}\left(\omega+\omega_{o}\right)= \\
\frac{2 \Gamma k_{B} T}{m}\left[\frac{1}{\left[\omega\left(\omega-2 \omega_{o}\right)\right]^{2}+\Gamma^{2}\left(\omega-\omega_{o}\right)^{2}}\right. \\
\left.+\frac{1}{\left[\omega\left(\omega+2 \omega_{o}\right)\right]^{2}+\Gamma^{2}\left(\omega+\omega_{o}\right)^{2}}\right] .
\end{aligned}
$$

We assume that $\omega \ll \omega_{o}$ and use the following since we are interested in the above PSD only at low frequencies

$$
\omega \pm 2 \omega_{o} \approx \pm 2 \omega_{o}, \quad \omega \pm \omega_{o} \approx \pm \omega_{o}
$$

The above approximations are similar to the ones in (34) that we employed in simplifying the deterministic dynamics. With (56), (55) can be simplified as follows

$$
\mathcal{S}_{r}\left(\omega-\omega_{o}\right)+\mathcal{S}_{r}\left(\omega+\omega_{o}\right) \approx \frac{4 k_{B} T}{m \Gamma \omega_{o}^{2}} \frac{1}{1+\left(\omega \tau_{r}\right)^{2}} .
$$

We substitute (57) above into (53) to obtain

$$
\begin{aligned}
\mathcal{S}_{d R e}(\omega) & =\mathcal{S}_{d I m}(\omega) \\
& =\frac{A_{o}^{2}}{4}\left|H_{L}(j \omega)\right|^{2} \frac{4 k_{B} T}{m \Gamma \omega_{o}^{2}} \frac{1}{1+\left(\omega \tau_{r}\right)^{2}} .
\end{aligned}
$$

We observe that

$$
\frac{1}{1+\left(\omega \tau_{r}\right)^{2}}=\left|H_{R}(j \omega)\right|^{2}
$$

with $H_{R}(\mathrm{~s})$ defined as in (43). Thus,

$$
\mathcal{S}_{d R e}(\omega)=\mathcal{S}_{d I m}(\omega)=\frac{A_{o}^{2} k_{B} T}{m \Gamma \omega_{o}^{2}}\left|H_{L}(j \omega)\right|^{2}\left|H_{R}(j \omega)\right|^{2} .
$$

Final stage in the demodulator is the $\arctan (\cdot)$ block, a memoryless nonlinearity. Up till now, we assumed that the resonator-demodulator chain is driven by only the resonator noise source. To correctly evaluate the effect of $\arctan (\cdot)$, we need to also consider the signal input to the resonator that is fed from the $\mathrm{CO}$ output. With $\mathrm{CO}$ output set to

$$
s_{o}(t)=A_{o} \cos \left(\omega_{o} t\right)=\frac{A_{o}}{2}\left[e^{j \omega_{o} t}+e^{-j \omega_{o} t}\right],
$$

the deterministic parts of the in-phase/quadrature signals at the inputs of $\arctan (\cdot)$ are the real/imaginary parts of

$$
\frac{A_{o}^{2}}{2} \frac{Q}{m \omega_{o}^{2}} e^{-j \frac{\pi}{2}}=0-j \frac{A_{o}^{2}}{2} \frac{Q}{m \omega_{o}^{2}}
$$

based on (18) and (38). The output $\theta_{d}(t)$ of $\arctan (\cdot)$ can be computed as follows

$$
\theta_{d}(t)=\arctan \frac{-\frac{A_{o}^{2}}{2} \frac{Q}{m \omega_{o}^{2}}+s_{d I m}(t)}{s_{d R e}(t)}
$$

where $s_{d R e}(t)$ and $s_{d I m}(t)$ are the noise signals at the inputs of $\arctan (\cdot)$. The noise signal $s_{d I m}(t)$ is much smaller than the DC signal term $-\frac{A_{o}^{2}}{2} \frac{Q}{m \omega_{o}^{2}}$. Thus,

$$
\theta_{d}(t) \approx \arctan \frac{-\frac{A_{o}^{2}}{2} \frac{Q}{m \omega_{o}^{2}}}{s_{d R e}(t)}
$$

Furthermore, the noise term $s_{d R e}(t)$ is also small. Thus, we use the following first-order Taylor's series expansion

$$
\arctan \frac{a}{x} \approx-\frac{\pi}{2}-\frac{x}{a} \text { for small } x
$$

to obtain

$$
\theta_{d}(t) \approx \arctan \frac{-\frac{A_{o}^{2}}{2} \frac{Q}{m \omega_{o}^{2}}}{s_{d R e}(t)} \approx-\frac{\pi}{2}+\frac{2 m \omega_{o}^{2}}{A_{o}^{2} Q} s_{d R e}(t)
$$

With the subtraction of the phase set point, i.e., $-\pi / 2$ at the output of the demodulator, the phase error $\theta_{e}(t)$ is given by

$$
\theta_{e}(t)=\theta_{d}(t)-\left(-\frac{\pi}{2}\right)=\frac{2 m \omega_{o}^{2}}{A_{o}^{2} Q} s_{d R e}(t) .
$$

The PSD of $\theta_{e}(t)$ can be computed based on (60) and (67):

$$
\mathcal{S}_{\theta_{e}}(\omega)=\frac{4 m \omega_{o}^{2} k_{B} T}{A_{o}^{2} Q^{2} \Gamma}\left|H_{L}(j \omega)\right|^{2}\left|H_{R}(j \omega)\right|^{2} .
$$

We use (37) in (68) and define

$$
\mathcal{S}_{\theta_{n}}(\omega)=\frac{4 m \omega_{o}^{2} k_{B} T}{A_{o}^{2} Q^{2} \Gamma}=\frac{2 m \tau_{r} \omega_{o}^{2} k_{B} T}{A_{o}^{2} Q^{2}}
$$

as the PSD of a white noise process $\theta_{n}(t)$, which represents the thermomechanical noise of the resonator, in an inputreferred manner, in the model in Fig. 3.

\section{FLL Noise Analysis}

The closed-loop system is governed by the following equation, written directly in the Laplace domain, by going around the loop in Fig. 3:

$\Theta_{o}(\mathrm{~s})=\frac{1}{\mathrm{~s}} H_{P I}(\mathrm{~s}) H_{L}(\mathrm{~s})\left[H_{R}(\mathrm{~s})\left[\Theta_{n}(\mathrm{~s})+\Theta_{o}(\mathrm{~s})\right]-\Theta_{o}(\mathrm{~s})\right]$. 
We compute the transfer function from $\Theta_{n}(\mathrm{~s})$ to $\Theta_{o}(\mathrm{~s})$ by solving this equation, and substitute (20), (43) into the result:

$$
\begin{aligned}
H_{\theta_{n}}^{\theta_{o}}(\mathrm{~s})=\frac{\Theta_{o}(\mathrm{~s})}{\Theta_{n}(\mathrm{~s})} & =\frac{H_{P I}(\mathrm{~s}) H_{L}(\mathrm{~s}) H_{R}(\mathrm{~s})}{\mathrm{s}+H_{P I}(\mathrm{~s}) H_{L}(\mathrm{~s})\left[1-H_{R}(\mathrm{~s})\right]} \\
& =\frac{\left(K_{p}+\frac{K_{i}}{\mathrm{~s}}\right) H_{L}(\mathrm{~s}) \frac{1}{1+\mathrm{s} \tau_{r}}}{\mathrm{~s}+\left(K_{p}+\frac{K_{i}}{\mathrm{~s}}\right) H_{L}(\mathrm{~s}) \frac{\mathrm{s} \tau_{r}}{1+\mathrm{s} \tau_{r}}} .
\end{aligned}
$$

We manipulate the above expression to obtain

$$
H_{\theta_{n}}^{\theta_{o}}(\mathrm{~s})=\frac{1}{\mathrm{~s}} \frac{1}{\tau_{r}}\left[\frac{\left(\mathrm{s} K_{p}+K_{i}\right) H_{L}(\mathrm{~s})}{\mathrm{s}^{2}+\frac{\mathrm{s}}{\tau_{r}}+\left(\mathrm{s} K_{p}+K_{i}\right) H_{L}(\mathrm{~s})}\right] .
$$

Due to (22), the transfer function from $\Theta_{n}(\mathrm{~s})$ to the $\mathrm{CO}$ frequency deviation $\Delta \Omega(\mathrm{s})$ is

$$
H_{\theta_{n}}^{\Delta \Omega}(\mathrm{s})=\frac{1}{\tau_{r}}\left[\frac{\left(\mathrm{s} K_{p}+K_{i}\right) H_{L}(\mathrm{~s})}{\mathrm{s}^{2}+\frac{\mathrm{s}}{\tau_{r}}+\left(\mathrm{s} K_{p}+K_{i}\right) H_{L}(\mathrm{~s})}\right] .
$$

We can then compute the PSD of $\Delta \Omega(t)$ as follows

$$
\mathcal{S}_{\Delta \Omega}(\omega)=\left|H_{\theta_{n}}^{\Delta \Omega}(j \omega)\right|^{2} \mathcal{S}_{\theta_{n}}(\omega)
$$

with $\mathcal{S}_{\theta_{n}}(\omega)$ in (69). The transfer function in (72) satisfies

$$
H_{\theta_{n}}^{\Delta \Omega}(\mathrm{s} \rightarrow 0)=\frac{1}{\tau_{r}}, \quad H_{\theta_{n}}^{\Delta \Omega}(\mathrm{s} \rightarrow \pm \infty)=0
$$

with an appropriate low-pass filter $H_{L}(\mathrm{~s})$ in the demodulator. That is, $H_{\theta_{n}}^{\Delta \Omega}$ (s) represents a low-pass filter, with a bandwidth that is essentially the loop bandwidth for the FLL.

\section{Characterizing FLL Performance via Allan Deviation}

Allan Deviation [30]-[34] is the standard measure of frequency stability [42], widely used for resonant sensors. We define the fractional frequency deviation

$$
y(t)=\frac{\Delta \Omega(t)}{\omega_{o}}
$$

where $\Delta \Omega(t)$ is the frequency deviation and $\omega_{o}$ is the nominal $\mathrm{CO}$ frequency. The averaged fractional frequency deviation is defined as

$$
\bar{y}(t, \tau)=\frac{1}{\tau} \int_{0}^{\tau} y(t+u) d u
$$

where $\tau$ is the averaging time. The $i$ th sample of $\bar{y}(t, \tau)$ is given by

$$
\bar{y}_{i}=\bar{y}(i \tau, \tau)
$$

where the sampling interval is chosen to be equal to the averaging time $\tau$. Allan Variance is computed as follows

$$
\sigma_{y}^{2}(\tau)=\frac{1}{2} \mathbf{E}\left[\left(\bar{y}_{i+1}-\bar{y}_{i}\right)^{2}\right]
$$

with Allan Deviation $\sigma_{y}(\tau)=\sqrt{\sigma_{y}^{2}(\tau)}$. The above implicitly assumes that $\sigma_{y}^{2}(\tau)$ is a function of only the averaging time $\tau$, independent of the sampling times represented by $i$ and $i+1$. This is so when $y(t)$ is a stationary process: Frequency deviation $\Delta \Omega(t)$ is indeed stationary, as the output of a stable, linear and time-invariant system (with transfer function
$H_{\theta_{n}}^{\Delta \Omega}(\mathrm{s})$ in (72)) with its input set to white, stationary noise $\theta_{n}(t)$. It can be shown that [31], [33]

$$
\sigma_{y}^{2}(\tau)=\frac{4}{\pi \tau^{2}} \int_{-\infty}^{+\infty} \frac{\left[\sin \left(\frac{\omega \tau}{2}\right)\right]^{4}}{\omega^{2}} \mathcal{S}_{y}(\omega) d \omega
$$

where $\mathcal{S}_{y}(\omega)$ is the PSD of $y(t)$ given by

$$
\mathcal{S}_{y}(\omega)=\frac{1}{\omega_{o}^{2}} \mathcal{S}_{\Delta \Omega}(\omega)
$$

due to (75) with $\mathcal{S}_{\Delta \Omega}(\omega)$ in (73). With the transfer function in (72), it is not possible to evaluate the Allan Variance integral in (79) analytically. However, we can evaluate it numerically for all values of $\tau$, for any choice of the low-pass filter $H_{L}$ (s) and the controller parameters $K_{p}$ and $K_{i}$. On the other hand, it is really desirable that we have an analytical handle on the frequency tracking accuracy of the FLL system: We evaluate the integral in (79) analytically, for values of $\tau$ that are larger than the loop time constant, thus computing a large- $\tau$ asymptote for the Allan Deviation. This result will be practically valuable, since the FLL is able to track the frequency deviations within its bandwidth, or in other words, at time scales that are longer than the loop time constant. Frequency deviations that occur faster than the loop time constant are attenuated by the loop dynamics, rendering the FLL tracking system not useful at these short time scales.

The large- $\tau$ asymptote for $\sigma_{y}^{2}(\tau)$ in (79) can be computed by approximating the low-pass PSD $\mathcal{S}_{y}(\omega)$ with its value at zero (low) frequency, i.e., with

$$
\mathcal{S}_{y}(\omega) \approx \mathcal{S}_{y}(0)=\frac{1}{\omega_{o}^{2}} \mathcal{S}_{\Delta \Omega}(0)=\frac{\mathcal{S}_{\theta_{n}}(\omega)}{\omega_{o}^{2} \tau_{r}^{2}}=\frac{\mathcal{S}_{\theta_{n}}}{\omega_{o}^{2} \tau_{r}^{2}}
$$

where we used (73) and (74), with $\mathcal{S}_{\theta_{n}}(\omega)=\mathcal{S}_{\theta_{n}}$ as in (69). We substitute (81) into (79) and evaluate the integral to obtain

$$
\begin{aligned}
\sigma_{y}^{2}(\tau) & =\frac{4}{\pi \tau^{2}} \int_{-\infty}^{+\infty} \frac{\left[\sin \left(\frac{\omega \tau}{2}\right)\right]^{4}}{\omega^{2}} \frac{\mathcal{S}_{\theta_{n}}}{\omega_{o}^{2} \tau_{r}^{2}} d \omega \\
& =\frac{\mathcal{S}_{\theta_{n}}}{\omega_{o}^{2} \tau_{r}^{2}} \frac{1}{\tau} .
\end{aligned}
$$

$\tau_{r}$ above is the resonator time constant defined by (37), whereas $\tau$ is the averaging time used in the definition of Allan Variance. The above result for $\sigma_{y}^{2}(\tau)$ is valid for large $\tau$, larger than the loop time constant. We use (69) and (37) in (82):

$$
\sigma_{y}^{2}(\tau)=\frac{m \omega_{o} k_{B} T}{A_{o}^{2} Q^{3}} \frac{1}{\tau} \text { for large } \tau .
$$

The factor (that multiplies $1 / \tau$ ) above is expressed in terms of $m, \omega_{o}, Q, k_{B}, T$, and the amplitude $A_{o}$ of the signal that drives the resonator. We express this factor in terms of a Signal-toNoise-Ratio (SNR) for the resonator, defined as follows

$$
\begin{aligned}
\text { SNR } & =\sqrt{\frac{\text { signal power at resonator input }}{\text { noise power at resonator input }}} \\
& =\sqrt{\frac{A_{o}^{2} / 2}{\mathcal{S}_{t h m} \mathrm{BW} 2}}=\sqrt{\frac{A_{o}^{2} Q}{8 m \omega_{o} k_{B} T \mathrm{BW}}} .
\end{aligned}
$$

$\mathcal{S}_{t h m}$ is the input-referred, white (two-sided) PSD of the thermomechanical noise given in (2). BW is defined as the 
(one-sided, hence the factor of 2) noise bandwidth. BW is typically set to the bandwidth of the low-pass filters in the demodulator. Alternatively, it could be set to the FLL loop bandwidth. The particular choice for BW simply affects the SNR definition, there is nothing fundamental about it. We have the following relationship for the product of $Q$ and SNR:

$$
Q \mathrm{SNR}=\sqrt{\frac{A_{o}^{2} Q^{3}}{8 m \omega_{o} k_{B} T \mathrm{BW}}} .
$$

Allan Variance in (83) can be expressed as

$$
\sigma_{y}^{2}(\tau)=\frac{m \omega_{o} k_{B} T}{A_{o}^{2} Q^{3}} \frac{1}{\tau}=\frac{1}{8(Q \mathrm{SNR})^{2} \mathrm{BW}} \frac{1}{\tau}
$$

and Allan Deviation is

$$
\sigma_{y}(\tau)=\frac{1}{2 \sqrt{2} Q \operatorname{SNR} \sqrt{\mathrm{BW}}} \frac{1}{\sqrt{\tau}} .
$$

\section{Results And Discussion}

\section{A. Setup and System Parameters}

The FLL bandwidth is typically set to a small fraction of the resonance frequency, limited by the capabilities of the loop components. We choose $K_{p}$ and $K_{i}$, as suggested in [6],

$$
K_{p}=\omega_{\mathrm{FLL}}, \quad K_{i}=\frac{\omega_{\mathrm{FLL}}}{\tau_{r}}
$$

where $\omega_{\mathrm{FLL}}$ is the desired loop bandwidth. If we substitute equation (88) into equation (72), a pole-zero cancellation [6] occurs, and simplifies to

$$
H_{\theta_{n}}^{\Delta \Omega}(\mathrm{s})=\frac{1}{\tau_{r}}\left[\frac{H_{L}(\mathrm{~s})}{H_{L}(\mathrm{~s})+\frac{\mathrm{s}}{\omega_{\mathrm{FLL}}}}\right] .
$$

The bandwidth of the filters in the demodulator is typically larger than $\omega_{\mathrm{FLL}}$, implying $H_{L}\left(j \omega_{\mathrm{FLL}}\right) \approx 1$. Hence, we have

$$
H_{\theta_{n}}^{\Delta \Omega}(\mathrm{s}) \approx \frac{1}{\tau_{r}} \begin{cases}\frac{1}{1+\frac{\mathrm{s}}{\omega_{\mathrm{FLL}}}} & \text { for }|\mathrm{s}| \leq \omega_{\mathrm{FLL}} \\ \frac{\omega_{\mathrm{FLL}} H_{L}}{\mathrm{~s}}(\mathrm{~s}) & \text { for }|\mathrm{s}| \gg \omega_{\mathrm{FLL}}\end{cases}
$$

with an effective first-order loop dynamics. Resonator and system parameters are chosen similar to the ones in [7]. FLL bandwidth is $\omega_{\mathrm{FLL}}=5 \times 10^{-5} \omega_{r}$. Hence, the loop time-constant is $2 \times 10^{4}$ periods of the high-frequency resonator signal. The low-pass filters in the demodulator are $4 t h$ order Butterworth filters with passband edge frequency $\omega_{L}=8 \omega_{\mathrm{FLL}}$. We choose $\mathrm{BW}=\omega_{L}=8 \omega_{\mathrm{FLL}}$ used in the SNR definition in (84).

We define dynamic range $\mathrm{DR}$ for the resonator with $\mathrm{DR}=$ $20 \log _{10}$ (SNR). We present results for two cases, a low quality factor, $Q=50$, and a high one, $Q=10000$, with the same resonance frequency. To compare these two cases at the onset of Duffing nonlinearity, we adjust the drive strength in such a way so that $\mathrm{SNR} \propto 1 / Q$ [7]. That is, we consider two cases, (i) high $Q$ with low $\mathrm{DR}$, and (ii) low $Q$ with high $\mathrm{DR}$, as in [7]. We note that SNR in (84) seems to be proportional to $\sqrt{Q}$. This is the case only if the amplitude $A_{o}$ of the signal that drives the resonator is held constant as $Q$ changes. As it was done in [7], we adjust the drive amplitude $A_{o}$ in such a way so that the resonator operates at the end of its linear range, i.e., at the onset of Duffing nonlinearity. The critical resonator output amplitude at the onset of Duffing nonlinearity is known to have an inverse square root dependence on $Q$ [7], [43]. The corresponding (input) drive amplitude required to keep the resonator at this critical point is then proportional to $1 /(Q \sqrt{Q})$, since the resonator transfer function from its input to output is proportional to $Q$ as seen in (38). If the drive amplitude is adjusted as prescribed as $Q$ changes, then we have SNR $\propto 1 / Q$. We choose DR $=20 \log _{10}(\mathrm{SNR})$ at the Duffing limit, by setting it to $62 \mathrm{~dB}$ for $Q=8286$ as it was the case in the experiments reported in [7, Fig. 4]. For other $Q$ values, we adjust DR so that SNR $\propto 1 / Q$. Thus, we have $\mathrm{DR} \approx 60$ (106) $\mathrm{dB}$ for $Q=10000(50)$.

\section{B. Results and Comparison With Stochastic Simulations}

In Fig. 5(a), we present results for Allan Deviation (AD), and in Fig. 5(b) for the PSD of fractional frequency deviation, for the two cases considered, (i) $Q=50, \mathrm{DR} \approx 106 \mathrm{~dB}$, (ii) $Q=10000, \mathrm{DR} \approx 60 \mathrm{~dB}$, based on both theory and also stochastic simulations (Appendix B). It is noteworthy that there is excellent agreement between the analytical results and the ones obtained from simulations, even though there is no free parameter in the model. Discrepancies at larger $\tau$ are expected for $A D$, due to the inaccuracy of estimation from time-limited simulation data. The agreement at low $\tau$, below and exceeding the loop time-constant, is excellent.

With $\mathrm{SNR} \propto 1 / Q, \mathrm{AD}$ is independent of $Q$ for all values of $\tau$. The results presented in Fig. 5 for the two cases considered fall exactly on top of each other, due to the scaling factor in equation (87) and the choice for the controller parameters in equation (88). That is, there is no difference in performance when the two cases are compared.

The particular choice of the controller parameters in equation (88) is unique in the sense that it results in a pole-zero cancellation [6] in the transfer function $H_{\theta_{n}}^{\Delta \Omega}$ (s), making its remaining poles and zeros independent of $Q$. Thus, AD is then independent of $Q$ for all values of $\tau$, when SNR is adjusted so as to hold $Q$ SNR constant, as in the two cases considered in Fig. 5. On the other hand, if the controller parameters are not chosen as in equation (88), or if another type of controller is used, then $A D$ will not necessarily be independent of $Q$ for all values of $\tau$, even when $Q$ SNR is held constant. However, the large- $\tau$ asymptote (for values of $\tau$ larger than the loop time-constant) will always be given by equation (87), i.e., independent of $Q$ with constant $Q$ SNR. In order to illustrate this, we show, in Fig. 6, AD for three different parameter choices, $K_{i}^{1}=\frac{\omega_{\mathrm{FLL}}}{\tau_{r}}$ (as in equation (88)), $K_{i}^{2}=\frac{\omega_{\mathrm{FLL}}}{3 \tau_{r}}$, and $K_{i}^{3}=\frac{3 \omega_{\mathrm{FLL}}}{\tau_{r}}$. All other parameters were chosen as described before. As it can be observed in Fig. 6, the controller design has an effect on AD only for low values of $\tau$, at or below the loop time-constant. However, this is not so important from a practical point of view: The FLL system is useful in tracking frequency deviations only at time scales longer than the loop time-constant, since it can not react to faster changes. Thus, the essential conclusions stated above do not change at different feedback parameters, i.e., one obtains 

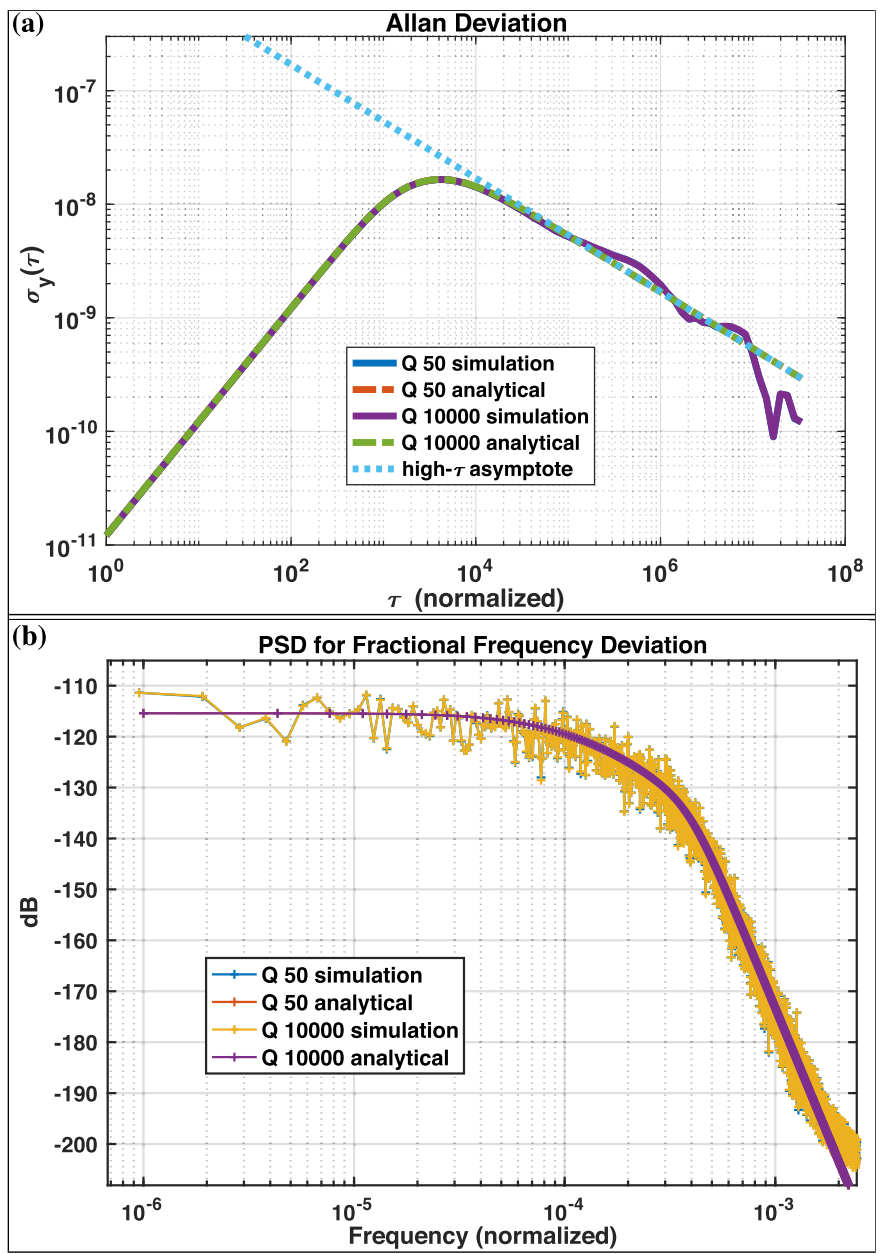

Fig. 5. (a) AV integral in equation (79) was evaluated numerically. The $\tau$ axis is normalized (number of cycles of the resonator output). The large- $\tau$ asymptote in equation (83) coincides with the results for $\tau>$ $10^{5}$. The loop time-constant is $2 \times 10^{4}$ (normalized). (b) The analytical results were obtained by evaluating equations (69), (72) and (73). The frequency axis is normalized with the resonance frequency. The PSD of frequency deviation has a Lorentzian shape, up to and exceeding the loop bandwidth, as predicted by equation (90). For larger frequencies, PSD exhibits a faster roll-off due to the effect of the higher order lowpass filters in the demodulator. The completely smooth curves in the graphs are based on theory, whereas the jagged ones (for large $\tau$ in the case of $A D$ ) are estimated from stochastic simulations as described in Appendix B. The two curves based on theory (as well as simulations) for the two $Q$ values considered fall on top of each other, and hence impossible to distinguish. There is a slight discrepancy between theory and simulations for large $\tau$ for $A D$, due to the inaccuracy of estimation for time-limited simulation data.

the same relevant performance in the two cases considered, (i) high $Q$ with low $\mathrm{DR}$, and (ii) low $Q$ with high DR.

\section{Frequency Step Deviation as a New Performance Metric}

The results in Fig. 6 should be interpreted considering the response of the FLL to resonance frequency shifts induced by events of interest, e.g., addition of mass. In fact, $A D$ alone is not an adequate performance measure for characterizing an FLL based resonator tracking system. We propose a new criterion called FSTDEV (Frequency STep DEViation) that combines AD with frequency step response (FSTR) as a better

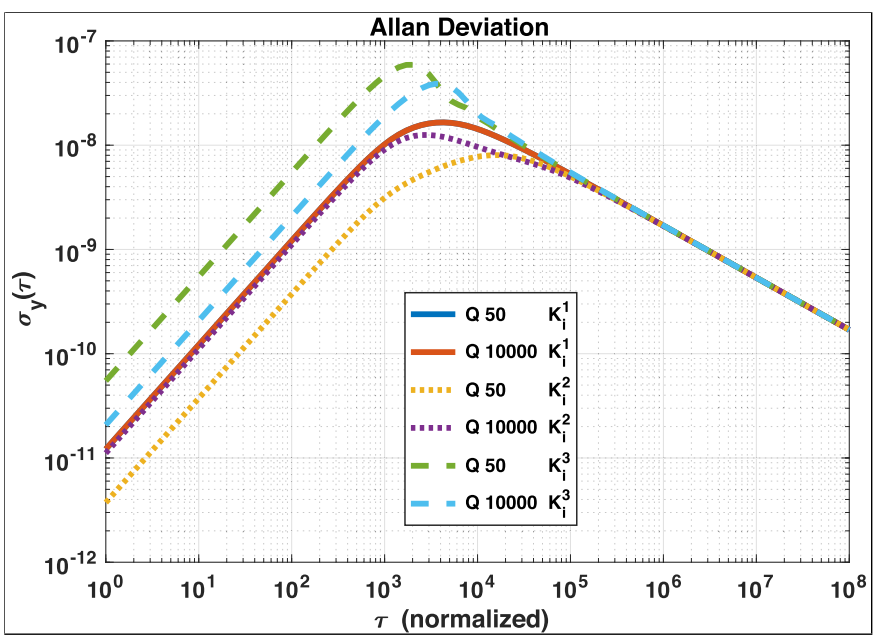

Fig. 6. $\mathrm{AD}$ versus $K_{j}$. As controller parameters change, large- $\tau$ asymptotes overlap as expected.

performance measure for an FLL system

$$
\operatorname{FSTDEV}(\tau) \triangleq \sigma_{y}^{F L L} \sqrt{\left[1-f_{s t r}(\tau)\right]^{2}+\left[\frac{\sigma_{y}(\tau)}{\sigma_{y}^{F L L}}\right]^{2}}
$$

where $f_{\text {str }}(\tau)$ is FSTR of the FLL (normalized so that $f_{\text {str }}(0)=0$ and $\left.f_{\text {str }}(\tau \rightarrow \infty)=1\right), \sigma_{y}(\tau)$ is $\mathrm{AD}$, and $\sigma_{y}^{F L L}$ is the peak value of AD (occurring around when $\tau$ is equal to the loop time-constant, ignoring frequency drift due to thermal and other effects). The rationale behind the proposed FSTDEV (smaller the better, coinciding with AD for large $\tau$ exceeding the loop time-constant) is that, if a frequency measurement is performed, after an event of interest but before the FLL locks to the new resonance frequency, the transient frequency settling error needs to be considered in addition to the frequency fluctuations due to noise when quantifying frequency accuracy. FSTDEV incorporates closed-loop behavior due to both deterministic and noisy FLL dynamics in a self-consistent manner. Fig. 7 shows FSTDEV, AD and FSTR, leading to the following conclusions: (i) $\operatorname{FSTDEV}(\tau)$ improves as $\tau$ is increased, with the asymptote for large $\tau$ coinciding with $A D$ and indicating that FLL has achieved lock. (ii) FSTDEV for $K_{i}^{1}$ is the same for the two $Q$ values. This is due to the fact that, with $K_{i}^{1}$, the loop transfer function and the loop dynamics is independent of $Q$, as described before. (iii) If the choice of the controller parameters, as for $K_{i}^{3}$, results in an under-damped, ringing response, the performance of the sensor as quantified by FSTDEV worsens for time scales shorter than the loop time constant. (iv) FSTDEV is independent of the choice of the controller parameters for large $\tau$, i.e., once FSTR settles. (v) A faster responding loop, corresponding to a shorter settling time in FSTR, results in larger AD values within time scales shorter than the loop time constant. For $K_{i}^{2}$, the $Q=10000$ case, versus $Q=50$, results in a faster responding loop at the expense of increased $A D$, amounting to a worse overall performance as quantified by FSTDEV. (vi) For a given $Q$, controller parameters and hence the trade-off between FSTR and AD can be optimized to obtain a better performance at short time scales as quantified by FSTDEV. 


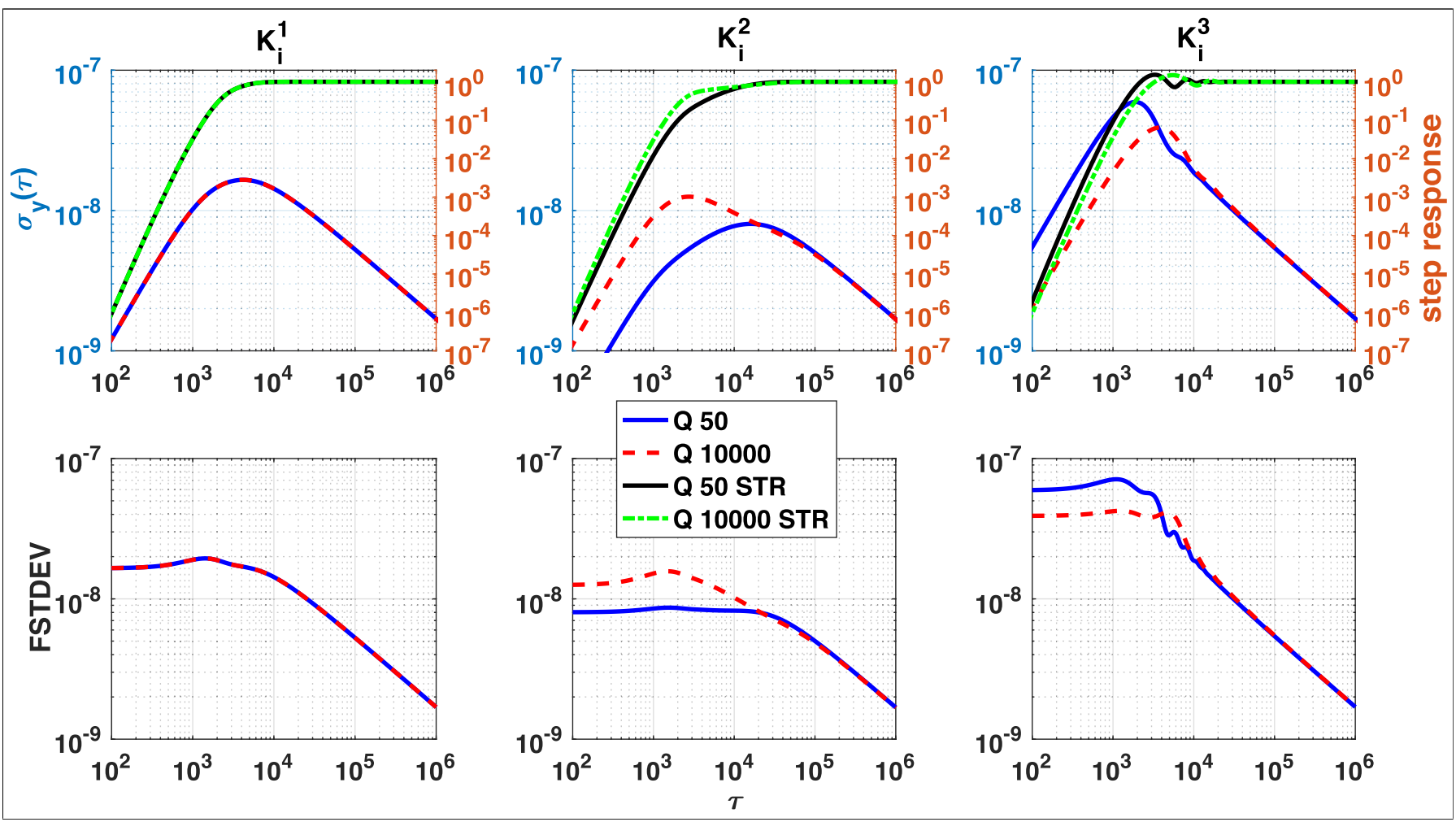

Fig. 7. AD (upper panes: left axis), Frequency Step Response (STR, upper panes: right axis) and FSTDEV (lower panes), versus $K_{i}$. The two curves for the two $Q$ values for $K_{i}^{1}$ case (in leftmost panes) fall exactly on top of each other, hence impossible to distinguish.

\section{Comparison With Previous Work and Discussion}

The results and theory presented in [7, Fig. 4 and equation 10], in the Duffing limited regime where drive strength is adjusted so that $\mathrm{SNR} \propto 1 / Q$, show that $\mathrm{AD}$ is proportional to $Q$. The results we have derived and reported are in stark contrast to these results presented by Roy et al. [7]. Our equation (87) and equation 10 in [7] are clearly different. The results derived from our theory, as captured in equation (87), show that $\mathrm{AD} \sigma_{y}(\tau)$ is inversely proportional to $Q \mathrm{SNR}$, for values of $\tau$ larger than the FLL loop time constant. That is, in the Duffing limited regime where SNR $\propto 1 / Q, \mathrm{AD} \sigma_{y}(\tau)$ is independent of $Q$ for values of $\tau$ larger than the FLL loop time constant. Furthermore, if the controller parameters are chosen as in (88), our theory shows that $\mathrm{AD} \sigma_{y}(\tau)$ is independent of $Q$ for all values of $\tau$, as shown in Fig. 5(a), again in the Duffing limited regime.

The flattening of the phase noise spectrum at low frequencies [7, Fig. 5 and equation 9], that leads to equation 10 in [7], is the basis of the claim in [7] that the sensor performance can be improved with larger damping. The theory developed by Roy et al. [7] does not consider the closed-loop dynamics of the FLL tracking system. Our theory and results, which resolve the closed-loop dynamics in full detail, show that there is no such flattening of the phase noise spectrum under feedback in an FLL. In fact, as phase deviation $\theta_{o}(t)$ is simply the integral of the frequency deviation $\Delta \Omega(t)$, and since $\Delta \Omega(t)$ has a near Lorentzian PSD as shown in Fig. 5(b), the phase spectrum $\left(1 / \omega^{2}\right.$ times the spectrum of $\Delta \Omega(t))$ does not flatten at low frequencies. On the contrary, the phase spectrum keeps increasing as frequency is lowered, a signature of random walk phase noise. Roy et al. [7] suggest that one can circumvent random walk phase noise in an FLL based system if a high precision signal generator is used. Our theory suggests otherwise. A high precision signal generator will produce negligible random walk phase noise arising from its internal noise sources, provided that it is controlled with a noiseless frequency control input. However, in an FLL, the frequency control signal is noisy, due to noise from other sources circulating around the loop and shaped by the loop dynamics. This is a fundamental aspect of FLL operation.

The theory we have developed supersedes the one presented in [7] as explained above. However, the experimental results presented in [7] which seem to corroborate their theory are then puzzling. We speculate that the resolution of this confusion may be in the invalidity of the assumption in [7] that the experimental setup used resolves thermomechanical noise above all other sources of noise. The experimental results presented in Fig. 4 in [7] show AD levels that are above $3 \times 10^{-8}$, whereas the results we present in Fig. 5(a) (based on application of our theory to a setup where the only noise in the system is of thermomechanical origin) show values for AD that are below $2 \times 10^{-8}$. Thus, it may be the case that there are other sources of noise that drown thermomechanical noise in the experiments reported in [7].

\section{CONCLUSION}

We presented a theory and noise analysis framework for FLL based resonant sensors, useful in deciphering the 
fundamental limitations and understanding basic trade-offs due to inherent noise arising from a number of sources. The framework we developed enables a firm analytical handle on the problem, resolving a major confusion in the literature. We considered a setting where the performance is limited by thermomechanical noise. In future work, we will extend the analysis framework to take into account other types of noise and nonideal dynamics in the resonator [10], electronic amplifier and instrumentation noise, fluctuations that arise from the actuation and sensing mechanisms in the mechanical, electrical and optical domains, non-negligible phase noise of the signal generator. Furthermore, we will develop extensions so that the analysis framework can be applied to a variety of FLL based sensor configurations, such as multi-mode mass spectrometry with multiple FLLs [3], and nonlinear trajectorylocked loop (TLL) [21] based sensing. Even though we have shown that lowering the quality factor of the resonator does not result in the claimed performance improvement, one may be able to obtain better performance by optimizing the controller and the filters in the demodulator in the presence of a variety of noise sources, that we plan to investigate in the near future using the proposed analysis framework.

\section{APPENDIX A \\ Spectral ChaRActerizations AND FiLTERING OF CYClO-STATIONARY PROCESSES}

Please see [39]-[41] for details regarding the spectral characterization and filtering of cyclo-stationary random processes. Our treatment below is based on [39]-[41]

Let $s(t)$ be a zero-mean, stationary Gaussian random process with the auto-correlation function

$$
R_{s}(\eta)=\mathbf{E}[s(t+\eta / 2) s(t-\eta / 2)]
$$

where $\mathbf{E}[\cdot]$ denotes the expectation operator. $R(\eta)$ is a function of only $\eta$, not $t$, due to the stationarity of the process. The PSD of $s(t)$ is defined as the Fourier transform of $R_{s}(\eta)$

$$
\mathcal{S}_{s}(\omega)=\mathcal{F}\left\{R_{S}(\eta)\right\} .
$$

Let $m(t)=A_{o} \cos \left(\omega_{o} t\right)$ be a modulating signal. We obtain the modulated random process $c(t)$ from $s(t)$ as follows

$$
c(t)=m(t) s(t)=A_{o} \cos \left(\omega_{o} t\right) s(t) .
$$

The auto-correlation function of $c(t)$ is a periodic function of $t$ and can be computed as follows

$$
\begin{aligned}
R_{c}(t, \eta) & =\mathbf{E}[c(t+\eta / 2) c(t-\eta / 2)] \\
& =m(t+\eta / 2) m(t-\eta / 2) R_{s}(\eta)
\end{aligned}
$$

where

$$
\begin{aligned}
m & (t+\eta / 2) m(t-\eta / 2) \\
& =A_{o}^{2} \cos \left(\omega_{o}(t+\eta / 2)\right) \cos \left(\omega_{o}(t-\eta / 2)\right) \\
& =\frac{A_{o}^{2}}{4}\left[e^{j \omega_{o} \eta}+e^{-j \omega_{o} \eta}+e^{j 2 \omega_{o} t}+e^{-j 2 \omega_{o} t}\right] .
\end{aligned}
$$

The PSD of $c(t)$ is a (periodic) function of $t$, in addition to $\omega$. The $t$ dependence is expanded into a Fourier series [39]-[41]

$$
\mathcal{S}_{c}(t, \omega)=\mathcal{F}\left\{R_{c}(t, \eta)\right\}=\sum_{k=-\infty}^{\infty} \mathcal{S}_{c}^{(k)}(\omega) e^{j k \omega_{o} t}
$$

where $\mathcal{S}_{c}^{(k)}(\omega)$ are called the cyclic spectra. In (97), the Fourier transform $\mathcal{F}\left\{R_{c}(t, \eta)\right\}$ is with respect to the variable $\eta$. For a stationary process, we have $\mathcal{S}_{c}^{(k)}(\omega)=0$ for $k \neq 0$. In this case, $\mathcal{S}_{c}^{(0)}(\omega)$ corresponds to the usual PSD for a stationary process. Using (95), (96) and (97), we obtain

$$
\begin{aligned}
& \mathcal{S}_{c}^{(0)}(\omega)=\frac{A_{o}^{2}}{4}\left[\mathcal{S}_{s}\left(\omega-\omega_{o}\right)+\mathcal{S}_{s}\left(\omega+\omega_{o}\right)\right] \\
& \mathcal{S}_{c}^{(2)}(\omega)=\mathcal{S}_{c}^{(-2)}(\omega)=\frac{A_{o}^{2}}{4} \mathcal{S}_{s}(\omega) \\
& \mathcal{S}_{c}^{(k)}(\omega)=0 \text { for all other } k .
\end{aligned}
$$

Next, we consider the (low-pass) filtering of $c(t)$ with a (linear and time-invariant) filter with frequency response $H_{L}(j \omega)$ [39], [40]. The output of the filter, $c_{L}(t)$, is in general a cyclo-stationary process. It can be shown that [41, eqn. 2.139] the cyclic spectra of $c_{L}(t)$ can be computed with

$$
\mathcal{S}_{c L}^{(k)}(\omega)=H_{L}\left(j \omega+j \frac{k \omega_{o}}{2}\right) \mathcal{S}_{c}^{(k)}(\omega) H_{L}^{*}\left(j \omega-j \frac{k \omega_{o}}{2}\right)
$$

where $*^{*}$ is complex-conjugate. The input cyclic spectra $\mathcal{S}_{c}^{(k)}(\omega)$ is nonzero only for $k=0, \pm 2$. We use (99) to obtain

$$
\begin{aligned}
\mathcal{S}_{c L}^{(0)}(\omega) & =\left|H_{L}(j \omega)\right|^{2} \mathcal{S}_{c}^{(0)}(\omega) \\
\mathcal{S}_{c L}^{(2)}(\omega) & =H_{L}\left(j\left(\omega+\omega_{o}\right)\right) \mathcal{S}_{c}^{(2)}(\omega) H_{L}^{*}\left(j\left(\omega-\omega_{o}\right)\right) \\
\mathcal{S}_{c L}^{(-2)}(\omega) & =H_{L}\left(j\left(\omega-\omega_{o}\right)\right) \mathcal{S}_{c}^{(-2)}(\omega) H_{L}^{*}\left(j\left(\omega+\omega_{o}\right)\right) \\
\mathcal{S}_{c L}^{(k)}(\omega) & =0 \text { for all other } k
\end{aligned}
$$

The result above is valid for any input stationary process $s(t)$ and for any filter $H_{L}(j \omega)$. We consider the case when $H_{L}(j \omega)$ is a low-pass filter with an effective bandwidth that is much less than $\omega_{o}$, satisfying $H_{L}\left( \pm j \omega_{o}\right) \approx H_{L}\left( \pm j 2 \omega_{o}\right) \approx 0$. Then,

$$
\begin{aligned}
& H_{L}\left(j\left(\omega-\omega_{o}\right)\right) \\
& \times H_{L}^{*}\left(j\left(\omega+\omega_{o}\right)\right)
\end{aligned}= \begin{cases}H_{L}\left(-j \omega_{o}\right) H_{L}^{*}\left(j \omega_{o}\right) \approx 0 & \omega \approx 0 \\
H_{L}(0) H_{L}^{*}\left(j 2 \omega_{o}\right) \approx 0 & \omega \approx \omega_{o} \\
H_{L}\left(-j 2 \omega_{o}\right) H_{L}^{*}(0) \approx 0 & \omega \approx-\omega_{o} .\end{cases}
$$

In fact, $H_{L}\left(j\left(\omega-\omega_{o}\right)\right) H_{L}^{*}\left(j\left(\omega+\omega_{o}\right)\right) \approx 0$ for all $\omega$. Then, based on (100), we conclude $\mathcal{S}_{c L}^{(k)}(\omega)=0$ for $k \neq 0$. That is, the output of the low-pass filter $H_{L}(j \omega)$ becomes a stationary process with PSD

$$
\begin{aligned}
\mathcal{S}_{c L}(\omega) & =\mathcal{S}_{c L}^{(0)}(\omega)=\left|H_{L}(j \omega)\right|^{2} \mathcal{S}_{c}^{(0)}(\omega) \\
& =\frac{A_{o}^{2}}{4}\left|H_{L}(j \omega)\right|^{2}\left[\mathcal{S}_{s}\left(\omega-\omega_{o}\right)+\mathcal{S}_{s}\left(\omega+\omega_{o}\right)\right] .
\end{aligned}
$$

Thus, the low-pass filter stationarizes the cyclo-stationary noise process $c(t)$ by removing the high-order cyclic components [40]. Furthermore, (101) reveals that there is noise folding in the frequency domain due to the modulation in (94) [40]. The noise components of $s(t)$ at frequencies $\omega-\omega_{o}$ and $\omega+\omega_{o}$ fold and both generate noise at $\omega$ in $c_{L}(t)$. The low-pass filter $H_{L}(j \omega)$ also removes any high-frequency noise components in $c_{L}(t)$, producing a low-pass noise PSD. 


\section{APPENDIX B \\ STOCHASTIC SIMULATOR}

In developing the theory in Section IV, we used several approximations and assumptions. Even though these are well founded and justified, we verified them by comparing our analytical results against the ones obtained from extensive, carefully crafted time-domain stochastic simulations of the FLL system. In these simulations, none of the mentioned approximations are used. The system is simulated with full, high-frequency, nonlinear and time-varying models for the resonator and the demodulator as shown in Fig. 2. The simulator is based on the numerical solution of coupled differential equations that are solved using an appropriate numerical technique based on time-discretization. The time step of the simulation is set to be a small fraction $(\approx 1 / 100)$ of the period of the high-frequency signal at the output of the resonator. The thermomechanical noise of the resonator is introduced into the simulation using a random number generator. The time series data produced by the simulation is post-processed to estimate the spectral densities of the signals of interest (using Welch's method [44]), as well as Allan Variance (using the overlapping estimator [34]). The duration of the simulation is set to be a large number, e.g. $10^{8}$, of the periods of the high-frequency signal at the output of the resonator, so that it is at least thousands of loop time constants long. Thus, the simulator has to take a very large number of time steps, e.g. $10^{10}$, requiring hours of CPU time.

\section{REFERENCES}

[1] J. Chaste, A. Eichler, J. Moser, G. Ceballos, R. Rurali, and A. Bachtold, "A nanomechanical mass sensor with yoctogram resolution," Nature Nanotechnol., vol. 7, no. 5, pp. 301-304, 2012.

[2] A. K. Naik, M. S. Hanay, W. K. Hiebert, X. L. Feng, and M. L. Roukes, "Towards single-molecule nanomechanical mass spectrometry," Nature Nanotechnol., vol. 4, no. 7, pp. 445-450, 2009.

[3] M. S. Hanay et al., "Single-protein nanomechanical mass spectrometry in real time," Nature Nanotechnol., vol. 7, no. 9, pp. 602-608, 2012.

[4] S. Dominguez-Medina et al., "Neutral mass spectrometry of virus capsids above 100 megadaltons with nanomechanical resonators," Science, vol. 362, no. 6417, pp. 918-922, 2018.

[5] A. H. Ghadimi et al., "Elastic strain engineering for ultralow mechanical dissipation," Science, vol. 360, pp. 764-768, May 2018.

[6] S. Olcum, N. Cermak, S. C. Wasserman, and S. R. Manalis, "Highspeed multiple-mode mass-sensing resolves dynamic nanoscale mass distributions," Nature Commun., vol. 6, May 2015, Art. no. 7070.

[7] S. K. Roy, V. T. K. Sauer, J. N. Westwood-Bachman, A. Venkatasubramanian, and W. K. Hiebert, "Improving mechanical sensor performance through larger damping," Science, vol. 360, no. 6394, 2018, Art. no. eaar5220.

[8] J. Kitching, "Chip-scale atomic devices," Appl. Phys. Rev., vol. 5, no. 3, 2018, Art. no. 031302.

[9] J. R. Vig and Y. Kim, "Noise in microelectromechanical system resonators," IEEE Trans. Ultrason., Ferroelectr., Freq. Control, vol. 46, no. 6, pp. 1558-1565, Nov. 1999.

[10] A. Cleland and M. Roukes, "Noise processes in nanomechanical resonators," J. Appl. Phys., vol. 92, no. 5, pp. 2758-2769, 2002.

[11] K. L. Ekinci, Y. T. Yang, and M. L. Roukes, "Ultimate limits to inertial mass sensing based upon nanoelectromechanical systems," J. Appl. Phys., vol. 95, no. 5, pp. 2682-2689, 2004.

[12] E. Gavartin, P. Verlot, and T. J. Kippenberg, "Stabilization of a linear nanomechanical oscillator to its thermodynamic limit," Nature Commun., vol. 4, Dec. 2013, Art. no. 2860

[13] M. Sansa et al., "Frequency fluctuations in silicon nanoresonators," Nature Nanotechnol., vol. 11, no. 6, pp. 552-558, Jun. 2016.

[14] E. Kenig et al., "Optimal operating points of oscillators using nonlinear resonators," Phys. Rev. E, Stat. Phys. Plasmas Fluids Relat. Interdiscip. Top., vol. 86, Nov. 2012, Art. no. 056207.
[15] L. G. Villanueva et al., "Surpassing fundamental limits of oscillators using nonlinear resonators," Phys. Rev. Lett., vol. 110, no. 17, Apr. 2013, Art. no. 177208.

[16] E. Kenig, M. C. Cross, J. Moehlis, and K. Wiesenfeld, "Phase noise of oscillators with unsaturated amplifiers," Phys. Rev. E, Stat. Phys. Plasmas Fluids Relat. Interdiscip. Top., vol. 88, no. 6, 2013, Art. no. 062922.

[17] A. Demir and M. S. Hanay, "Numerical analysis of multidomain systems: Coupled nonlinear PDEs and DAEs with noise," IEEE Trans. Comput.-Aided Design Integr. Circuits Syst., vol. 37, no. 7, pp. 1445-1458, Jul. 2018.

[18] J. J. Gagnepain, J. Uebersfeld, G. Goujon, and P. Handel, "Relation between 1/f noise and Q-factor in quartz resonators at room and low temperatures, first theoretical interpretation," in Proc. 35th Annu. Symp. Freq. Control, May 1981, pp. 476-483.

[19] W. P. Robins, Phase Noise in Signal Sources: Theory and Applications. Edison, NJ, USA: IET, 1984.

[20] T. E. Parker, "Characteristics and sources of phase noise in stable oscillators," in Proc. 41st Annu. Symp. Freq. Control, May 1987, pp. 99-110.

[21] M. Yuksel, E. Orhan, C. Yanik, A. B. Ari, A. Demir, and M. S. Hanay, "Nonlinear nanomechanical mass spectrometry at the single-nanoparticle level," Nano Lett., vol. 19, no. 6, pp. 3583-3589, 2019.

[22] M. Kelleci, H. Aydogmus, L. Aslanbas, S. O. Erbil, and M. S. Hanay, "Towards microwave imaging of cells," Lab Chip, vol. 18, no. 3, pp. 463-472, 2018.

[23] M. Nikolic-Jaric et al., "Microwave frequency sensor for detection of biological cells in microfluidic channels," Biomicrofluidics, vol. 3, no. 3, 2009, Art. no. 034103.

[24] T. Albrecht, P. Grütter, D. Horne, and D. Rugar, "Frequency modulation detection using high- $Q$ cantilevers for enhanced force microscope sensitivity," J. Appl. Phys., vol. 69, no. 2, pp. 668-673, 1991.

[25] K. Kobayashi, H. Yamada, and K. Matsushige, "Frequency noise in frequency modulation atomic force microscopy," Rev. Sci. Instrum., vol. 80, no. 4, 2009, Art. no. 043708.

[26] J. D. Adams, B. W. Erickson, J. Grossenbacher, J. Brugger, A. Nievergelt, and G. E. Fantner, "Harnessing the damping properties of materials for high-speed atomic force microscopy," Nature Nanotechnol. vol. 11, no. 2, pp. 147-151, 2016.

[27] M. Z. Baykara, "Noncontact atomic force microscopy for atomicscale characterization of material surfaces," in Surface Science Tools for Nanomaterials Characterization. Berlin, Germany: Springer, 2015, pp. 273-316.

[28] F. J. Giessibl, "The qplus sensor, a powerful core for the atomic force microscope," Rev. Sci. Instrum., vol. 90, no. 1, 2019, Art. no. 011101.

[29] B. D. Hauer, C. Doolin, K. S. D. Beach, and J. P. Davis, "A general procedure for thermomechanical calibration of nano/micro-mechanical resonators," Ann. Phys., vol. 339, pp. 181-207, Dec. 2013.

[30] D. W. Allan, "Statistics of atomic frequency standards," Proc. IEEE, vol. 54, no. 2, pp. 221-230, Feb. 1966.

[31] V. F. Kroupa, Frequency Stability: Fundamentals and Measurement. New York, NY, USA: IEEE Press, 1983.

[32] E. Rubiola, Phase Noise and Frequency Stability in Oscillators. Cambridge, U.K.: Cambridge Univ. Press, 2009.

[33] A. Makdissi, F. Vernotte, and E. De Clercq, "Stability variances: A filter approach," IEEE Trans. Ultrason., Ferroelectr., Freq. Control, vol. 57, no. 5, pp. 1011-1028, May 2010.

[34] Allan Variance-Wikipedia, The Free Encyclopedia, Wikimedia Found., San Francisco, CA, USA, 2019.

[35] M. V. Salapaka, H. S. Bergh, J. Lai, A. Majumdar, and E. McFarland, "Multi-mode noise analysis of cantilevers for scanning probe microscopy," J. Appl. Phys., vol. 81, no. 6, pp. 2480-2487, 1997.

[36] R. Bowley and M. Sanchez, Introductory Statistical Mechanics, vol. 40. Oxford, U.K.: Clarendon, 1999.

[37] S. Benedetto and E. Biglieri, Principles of Digital Transmission: With Wireless Applications. New York, NY, USA: Kluwer, 1999.

[38] B. Yurke, D. S. Greywall, A. N. Pargellis, and P. A. Busch, "Theory of amplifier-noise evasion in an oscillator employing a nonlinear resonator," Phys. Rev. A, Gen. Phys., vol. 51, no. 5, p. 4211, May 1995.

[39] W. Gardner, Introduction to Random Processes. New York, NY, USA: McGraw-Hill, 1990.

[40] J. Roychowdhury, D. Long, and P. Feldmann, "Cyclostationary noise analysis of large RF circuits with multitone excitations," IEEE J. SolidState Circuits, vol. 33, no. 3, pp. 324-336, Mar. 1998.

[41] A. Demir and A. Sangiovanni-Vincentelli, Analysis and Simulation of Noise in Nonlinear Electronic Circuits and Systems. Boston, MA, USA: Kluwer, 1998 
[42] IEEE Standard Definitions of Physical Quantities for Fundamental Frequency and Time Metrology_Random Instabilities, IEEE Standard 1139-2008, Feb. 2009.

[43] S. Schmid, L. G. Villanueva, and M. L. Roukes, Fundamentals of Nanomechanical Resonators, vol. 49. Cham, Switzerland: Springer, 2016.

[44] P. D. Welch, "The use of fast Fourier transform for the estimation of power spectra: A method based on time averaging over short, modified periodograms," IEEE Trans. Audio Electroacoust., vol. AU-15, no. 2, pp. 70-73, Jun. 1967.

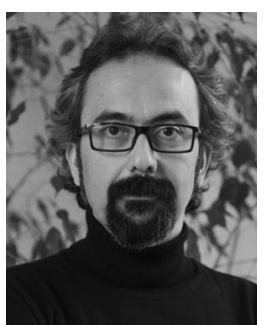

Alper Demir (F'12) received the B.S. degree from Bilkent University, Ankara, Turkey, in 1991, and the M.S. and Ph.D. degrees from the University of California at Berkeley, Berkeley, CA, USA, in 1994 and 1997, respectively. He was with Motorola, Austin, TX, USA; Cadence Design Systems, San Jose, CA, USA; Bell Laboratories, Murray Hill, NJ, USA; MIT, Cambridge, MA, USA; and the University of California at Berkeley. $\mathrm{He}$ has been a Faculty Member with Koç University, Istanbul, Turkey, since 2002. He was a recipient of the 2002 Best of ICCAD Award, the 2003/2014 IEEE/ACM William J. McCalla ICCAD Best Paper Awards, and the 2004 IEEE Guillemin-Cauer Award.

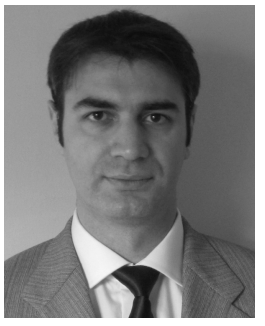

M. Selim Hanay received the B.S. degree from Sabancl University, Istanbul, Turkey, in 2003, and the Ph.D. degree from Caltech, Pasadena, CA, USA, in 2011. He was a Postdoctoral Research Fellow and a Research Scientist with Caltech. He is currently a Faculty Member with Bilkent University, Ankara, Turkey. His research on NEMS has been highlighted in the New York Times, IEEE Spectrum, Scientific American, Discover Magazine, and Slashdot. He was awarded the Gold Medal in the 31st International Chemistry Olympiads and a European Research Council Starting Investigator Grant. 